\title{
A classification of cohomology transfers for ramified covering maps
}

\author{
by \\ Marcelo A. Aguilar and Carlos Prieto (México, D.F.)
}

\begin{abstract}
We construct a cohomology transfer for $n$-fold ramified covering maps. Then we define a very general concept of transfer for ramified covering maps and prove a classification theorem for such transfers. This generalizes Roush's classification of transfers for $n$-fold ordinary covering maps. We characterize those representable cofunctors which admit a family of transfers for ramified covering maps that have two naturality properties, as well as normalization and stability. This is analogous to Roush's characterization theorem for the case of ordinary covering maps. Finally, we classify those families of transfers and construct some examples. In particular, we extend the determinant function in $\mathrm{GL}(k, \mathbb{C})$ to a transfer.
\end{abstract}

0. Introduction. In [3], we defined a transfer for ramified covering maps in ordinary cohomology. We start this paper by giving a transfer homomorphism $t^{p}: h(E)=[E, \mathcal{H}] \rightarrow h(X)=[X, \mathcal{H}]$ for any topological abelian monoid $\mathcal{H}$ and any ramified covering map $p: E \rightarrow X$. In particular, if $\mathcal{H}$ is an Eilenberg-Mac Lane space (modelled by a topological abelian group), then we have the cohomology transfer. This transfer is an example of what we shall call $(h, k)$-transfers, where $h$ and $k$ are representable cofunctors from the homotopy category of spaces to the category of sets, represented by spaces $\mathcal{H}$ and $\mathcal{K}$ (not necessarily topological abelian groups or $\mathrm{H}$-spaces). We use the properties of the transfer in ordinary cohomology to define the concept of a general $(h, k)$-transfer for ramified covering maps. We give a classification of these transfers that extends the classification of transfers for ordinary covering maps given by Roush [6]. In particular, the set of $(h, k)$-transfers has a canonical group structure when $k$ is group-valued. Our

2000 Mathematics Subject Classification: Primary 55R12, 57M12; Secondary 55Q05, $57 \mathrm{M} 10$.

Key words and phrases: transfer, covering maps, ramified covering maps, representable functors.

Research of C. Prieto was partially supported by PAPIIT grant IN110902 and by CONACYT grant 43724 . 
results are applied to the study of transfer families and their classification and to conclude that there are $(h, h)$-transfers if and only if $\mathcal{H}$ is a weak product of Eilenberg-Mac Lane spaces.

The structure of the paper is as follows. In Section 1 we recall the definition of a ramified covering map given by Smith [7] and define our $(h, h)$ transfer for $h(-)=[-, \mathcal{H}]$. In Section 2 we give the definition of a general $(h, k)$-transfer and study its properties. We prove that there is a one-to-one correspondence between $(h, k)$-transfers for $n$-fold ramified covering maps and elements in $k\left(\mathrm{SP}^{n} \mathcal{H}\right)$. We prove further that there are nontrivial transfers for $n$-fold ramified covering maps in singular cohomology (for large $n$ ) only when the dimensions of the cohomology groups are the same, and that those transfers are classified by the integers. In Section 3 we compare our transfers with transfers for ordinary covering maps and prove that our classification extends Roush's classification.

In Section 4 we consider families of $(h, k)$-transfers for $n$-fold ramified covering maps for all $n$ and give their classification. Namely, we prove that there is a one-to-one correspondence between families of $(h, k)$-transfers for ramified covering maps and elements in $\lim _{n} k\left(\mathrm{SP}^{n} \mathcal{H}\right)$. Analogously to Roush's characterization theorem for the case of ordinary covering maps, we give a characterization of those representable functors which admit a family of transfers. We also show that for singular cohomology, all transfers are determined by the transfers for 2-fold ramified covering maps. We finish the section by giving examples of transfers for functors that are not cohomology theories. In particular, we extend the determinant function det $: \operatorname{GL}(k, \mathbb{C}) \rightarrow \mathbb{C}^{*}$, which yields an element in $H^{2}(\operatorname{BGL}(k, \mathbb{C}))$, to a transfer for ramified covering maps $\tau$ : $\operatorname{Vect}_{k}^{\mathbb{C}}(-) \rightarrow \operatorname{Vect}_{1}^{\mathbb{C}}(-)$.

Finally, in Section 5, we study transfers for $h(-)=k(-)=H^{1}(-; \mathbb{Z})$ and prove that the transfers for ordinary covering maps are the same as those for ramified covering maps, i.e., in this case, one can extend in a unique way the transfers for ordinary covering maps to transfers for ramified covering maps. We conclude that for each $n$, the group of transfers for $n$-fold ramified covering maps in 1-cohomology is isomorphic to the group of transfers for ordinary covering maps, and both are isomorphic to $\mathbb{Z}$.

1. Transfers for $n$-fold ramified covering maps. We start by recalling L. Smith's definition of a ramified covering map (see [7]). We shall need the concept of $n$th symmetric power of $Y$ defined by

$$
\mathrm{SP}^{n} Y=\underbrace{Y \times \cdots \times Y}_{n} / \Sigma_{n}
$$

where $\Sigma_{n}$ represents the $n$th symmetric group acting on the product $Y \times \cdots \times Y$ by permuting coordinates. We denote the elements of $\operatorname{SP}^{n} Y$ by $\left\langle y_{1}, \ldots, y_{n}\right\rangle$. 
DeFinition 1.1. An $n$-fold ramified covering map is a continuous map $p: E \rightarrow X$ together with a multiplicity function $\mu: E \rightarrow \mathbb{N}$ such that the following hold:

(i) The fibers $p^{-1}(x)$ are finite (discrete), $x \in X$.

(ii) For each $x \in X, \sum_{e \in p^{-1}(x)} \mu(e)=n$.

(iii) The map $\varphi_{p}: X \rightarrow \mathrm{SP}^{n} E$ given by

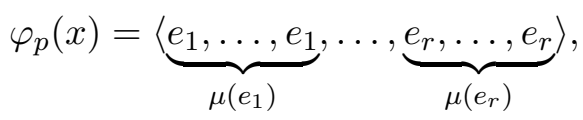

where $p^{-1}(x)=\left\{e_{1}, \ldots, e_{r}\right\}$, is continuous.

REMARK 1.2. Given an $n$-fold ramified covering map $p: E \rightarrow X$ with multiplicity function $\mu$, one can construct an $(n+1)$-fold ramified covering map as follows. Let $\bar{E}=E \sqcup X$ and $\bar{p}: \bar{E} \rightarrow X$ be such that $\left.\bar{p}\right|_{E}=p$ and $\left.\bar{p}\right|_{X}=\mathrm{id}_{X}$. Then $\bar{p}$ is a ramified covering map with the obvious multiplicity function. To see that $\bar{p}$ is indeed a ramified covering map, notice that $\varphi_{\bar{p}}$ is given by the following diagram:

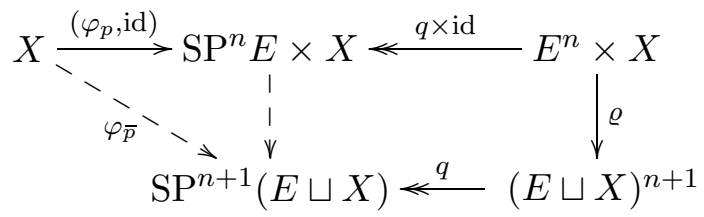

where $\varrho\left(\left(e_{1}, \ldots, e_{n}\right), x\right)=\left(e_{1}, \ldots, e_{n}, x\right)$. Observe that $q \times$ id is an identification, since $q$ is an open map. Therefore, $\varphi_{\bar{p}}$ is continuous.

On the other hand, given a map $f: Y \rightarrow X$, one can construct the induced $n$-fold ramified covering map $f^{*}(p): f^{*}(E) \rightarrow Y$ by taking the pullback $f^{*}(E)=\{(y, e) \in Y \times E \mid f(y)=p(e)\}$ and $f^{*}(p)=\operatorname{proj}_{Y}$. The induced multiplicity function $f^{*}(\mu): f^{*}(E) \rightarrow \mathbb{N}$ is given by $f^{*}(\mu)(y, e)$ $=\mu(e)$. Denote by $\widetilde{f}: f^{*}(E) \rightarrow E$ the projection $\operatorname{proj}_{E}$.

ExAMPLES 1.3. Typical examples of ramified covering maps are orbit maps $E \rightarrow E / G$ of actions of a finite group $G$ on a space $E$. They can be considered as $|G|$-fold ramified covering maps by taking $\mu(e)=\left|G_{e}\right|$, where $G_{e}$ denotes the isotropy subgroup of $e \in E$ and $|H|$ denotes the order of a group $H$.

It will be of particular interest to consider the following example. Let $B$ be a space and $\pi_{n}=\pi_{n}^{B}: B^{n} \times_{\Sigma_{n}} \bar{n} \rightarrow \mathrm{SP}^{n} B$ be given by $\pi_{n}\left\langle b_{1}, \ldots, b_{n} ; i\right\rangle$ $=\left\langle b_{1}, \ldots, b_{n}\right\rangle$, where $\bar{n}=\{1, \ldots, n\}$ and $\times_{\Sigma_{n}}$ represents the twisted product. Then $\pi_{n}$ is an $n$-fold ramified covering map with multiplicity function $\mu_{n}=\mu_{n}^{B}: B^{n} \times_{\Sigma_{n}} \bar{n} \rightarrow \mathbb{N}$ given by $\mu_{n}\left\langle b_{1}, \ldots, b_{n} ; i\right\rangle=\#\left\{j \mid b_{j}=b_{i}\right\}$ (see [7]). 
Definition 1.4. Let $p: E \rightarrow X$ be an $n$-fold ramified covering map with multiplicity function $\mu$. If $\mathcal{H}$ is a topological abelian group, define

$$
t^{p}:[E, \mathcal{H}] \rightarrow[X, \mathcal{H}] \quad \text { by } t^{p}([\widetilde{\alpha}])=[\alpha],
$$

where $\alpha(x)=\sum_{p(e)=x} \mu(e) \widetilde{\alpha}(e), x \in X$. To see that the map $\alpha$ is continuous and that its homotopy class depends only on the homotopy class of $\widetilde{\alpha}$, observe that $\alpha$ is given by the composite

$$
\alpha: X \stackrel{\varphi_{p}}{\rightarrow} \mathrm{SP}^{n} E \stackrel{\mathrm{SP}^{n} \widetilde{\alpha}}{\longrightarrow} \mathrm{SP}^{n} \mathcal{H} \rightarrow \mathcal{H},
$$

where the last map is given by the group structure on $\mathcal{H}$.

Let $X$ be a pointed space and $L$ be an abelian (topological) group. Denote by $F(X, L)$ the McCord topological group of functions $u: X \rightarrow L$ such that $u(*)=0$ and $u(x)=0$ for all but finitely many elements in $X$. This has the structure of a topological group (see [5] or [3]). If $\mathcal{H}$ is an Eilenberg-Mac Lane space of type $K(L, q)$, for instance given by $F\left(\mathbb{S}^{q}, L\right)$, then $t^{p}$ is the cohomology transfer

$$
t^{p}: \widetilde{H}^{q}(E ; L) \rightarrow \widetilde{H}^{q}(X ; L) .
$$

Here $\widetilde{H}^{q}$ stands for ordinary cohomology when the spaces involved have the homotopy type of CW-complexes, or for Čech cohomology if they are paracompact Hausdorff, provided that either $L$ is countable or the spaces are compactly generated (see [4]).

EXAMPLE 1.5. For the ramified covering map $\pi_{n}: B^{n} \times_{\Sigma_{n}} \bar{n} \rightarrow \mathrm{SP}^{n} B$ of 1.3 , the cohomology transfer is as follows. Let $\mathcal{H}$ be a topological abelian group. Then

$$
t^{\pi_{n}}:\left[B^{n} \times{ }_{\Sigma_{n}} \bar{n}, \mathcal{H}\right] \rightarrow\left[\mathrm{SP}^{n} B, \mathcal{H}\right]
$$

is given by $t^{\pi_{n}}([\widetilde{\alpha}])=[\alpha]$, where

$$
\alpha\left\langle b_{1}, \ldots, b_{n}\right\rangle=\sum_{i=1}^{n} \widetilde{\alpha}\left\langle b_{1}, \ldots, b_{n} ; i\right\rangle .
$$

The following propositions establish the fundamental properties of the transfer.

Proposition 1.6. If $p: E \rightarrow X$ is an $n$-fold ramified covering map, then the composite

$$
[X, \mathcal{F}] \stackrel{p^{*}}{\rightarrow}[E, \mathcal{F}] \stackrel{t^{p}}{\rightarrow}[X, \mathcal{F}]
$$

is multiplication by $n$.

Proof. If $[\alpha] \in[X, \mathcal{F}]$, then $t^{p} p^{*}(\alpha)=t^{p}(\alpha \circ p): X \rightarrow \mathcal{H}$, and

$$
t^{p}(\alpha \circ p)(x)=\sum_{p(e)=x} \mu(e) \alpha p(e)=\left(\sum_{p(e)=x} \mu(e)\right) \alpha(x)=n \cdot \alpha(x) .
$$

Thus $t^{p} p^{*}([\alpha])=n \cdot[\alpha]$. 
We also obtain the following.

Proposition 1.7. Let $\mathbb{Z}_{n}$ act on $X^{n}$ by cyclic permutation of coordinates, and take the quotient map $p: X^{n} \rightarrow X^{n} / \mathbb{Z}_{n}$. If the prime $q$ does not divide $n$, then $p^{*}: H^{l}\left(X^{n} / \mathbb{Z}_{n} ; \mathbb{Z}_{q}\right) \rightarrow H^{l}\left(X^{n} ; \mathbb{Z}_{q}\right)$ is a split monomorphism.

Proof. The map $p: X^{n} \rightarrow X^{n} / \mathbb{Z}_{n}$ is an $n$-fold ramified covering map. Take its transfer given by the additive structure of $K\left(\mathbb{Z}_{q}, l\right)$. Then $t^{p} \circ p^{*}$ is multiplication by $n$, thus an isomorphism. Hence $p^{*}$ is a split monomorphism.

The invariance under pullbacks is given by the following.

Proposition 1.8. If $p: E \rightarrow X$ is an $n$-fold ramified covering map, $\mathcal{F}$ is a topological abelian group, and $f: Y \rightarrow X$ is continuous, then the following diagram commutes:

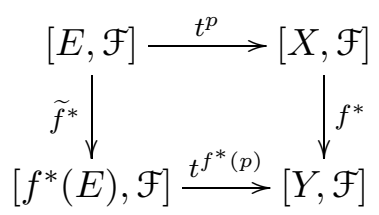

Proof. Let $\widetilde{\alpha}: E \rightarrow \mathcal{F}$ represent an element in $[E, \mathcal{F}]$. Then the map

$$
y \mapsto \sum_{f_{*}(p)(y, e)=y} f^{*}(\mu)(y, e) \widetilde{\alpha}(y, e)=\sum_{p(e)=f(y)} \mu(e) \widetilde{\alpha}(y, e),
$$

which represents $t^{f^{*}(p)} \widetilde{f}^{*}([\widetilde{\alpha}])$, clearly also represents $f^{*} t^{p}([\widetilde{\alpha}]) \in[Y, \mathcal{F}]$.

One further useful property of the cohomology transfer is the following.

Proposition 1.9. Let $f: B \rightarrow C$ be continuous and consider the commutative diagram

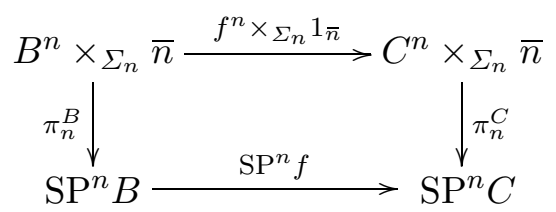

Then the following diagram commutes:

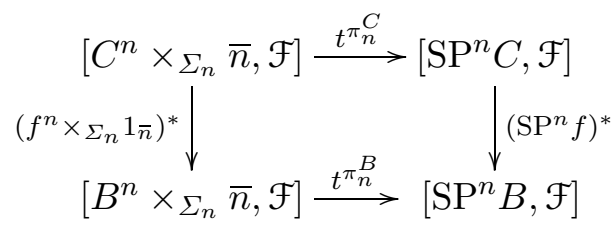

Proof. The result follows easily from the description of the transfer given in Example 1.5. 
In 1.6 we computed the composite $t^{p} \circ p^{*}$. The opposite composite $p^{*} \circ t^{p}$ is also interesting. An immediate calculation yields the following.

Proposition 1.11 (cf. $[3,5.6]$ ). Let $p: E \rightarrow X$ be an $n$-fold ramified covering map with multiplicity function $\mu$. Then the composite

$$
[E, \mathcal{F}] \stackrel{t^{p}}{\rightarrow}[X, \mathcal{F}] \stackrel{p^{*}}{\rightarrow}[E, \mathcal{F}]
$$

is given as follows. If $[\alpha] \in[E, \mathcal{F}]$, then $p^{*} t^{p}[\alpha]$ is represented by the map $\alpha^{\prime}: E \rightarrow \mathcal{F}$ given by

$$
\alpha^{\prime}(e)=\sum_{p\left(e^{\prime}\right)=p(e)} \mu\left(e^{\prime}\right) \varphi\left(e^{\prime}\right)
$$

In the case of an action of a finite group $G$ on $E$ and $X=E / G$, we have the following consequence.

Corollary 1.12 (cf. $[3,5.7]$ ). If $[\alpha] \in[E, \mathcal{F}]$, then $p^{*} t^{p}[\alpha]=\left[\alpha^{\prime}\right] \in[E, \mathcal{F}]$, where

$$
\alpha^{\prime}(e)=\sum_{g \in G} \alpha(g e)
$$

Proof. Just observe that the element $\alpha(g e)$ is repeated in the sum $\mu(e)=\left|G_{e}\right|$ times.

REMARK 1.13. Considering an action of $H$ on $E$ and a subgroup $K \subset H$, one has different ramified covering maps as depicted in

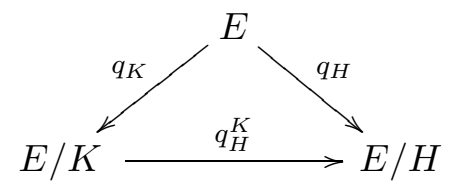

One may easily compute several combinations of the functions induced by these covering maps in homotopy sets and their transfers (see Propositions 1.15 and 2.11 below).

Another interesting property of the transfer is the relationship given by computing the transfer of the composition of two ramified covering maps. Before giving it we need the following.

Definition 1.14. Let $p: Y \rightarrow X$ be an $n$-fold ramified covering map with multiplicity function $\mu: Y \rightarrow \mathbb{N}$, and let $q: Z \rightarrow Y$ be an $m$ fold ramified covering map with multiplicity function $\nu: Z \rightarrow \mathbb{N}$. Then the composite $p \circ q: Z \rightarrow X$ is an $m n$-fold ramified covering map with multiplicity function $\xi: Z \rightarrow \mathbb{N}$ given by $\xi(z)=\nu(z) \mu(q(z))$. In order to verify that this composite is indeed an $m n$-fold ramified covering map, consider the wreath product $\Sigma_{n} 2 \Sigma_{m}$, defined as the semidirect product of $\Sigma_{n}$ and $\left(\Sigma_{m}\right)^{n}$, where $\Sigma_{n}$ acts on $\left(\Sigma_{m}\right)^{n}$ by permuting the $n$ factors. We have an action $\left(Z^{m} \times \cdots \times Z^{m}\right) \times \Sigma_{n} \imath \Sigma_{m} \rightarrow Z^{m} \times \cdots \times Z^{m}$ given by 
$\left(\zeta_{1}, \ldots, \zeta_{n}\right) \cdot\left(\sigma, \tau_{1}, \ldots, \tau_{n}\right)=\left(\zeta_{\sigma(1)} \cdot \tau_{1}, \ldots, \zeta_{\sigma(n)} \cdot \tau_{n}\right)$, where $\zeta_{i} \in Z^{m}$. Then we have the following diagram, where all maps are open:

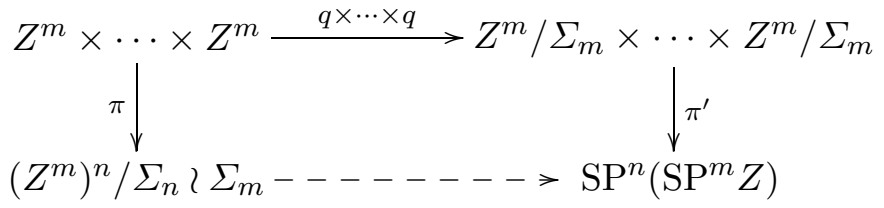

One may easily show that $\pi$ is compatible with $\pi^{\prime} \circ(q \times \cdots \times q)$. Therefore, there is a homeomorphism $X^{m n} / \Sigma_{n} \prec \Sigma_{m} \approx \mathrm{SP}^{n}\left(\mathrm{SP}^{m} Z\right)$ and hence one has a canonical quotient map $\varrho: \mathrm{SP}^{n}\left(\mathrm{SP}^{m} Z\right) \rightarrow \mathrm{SP}^{m n} Z$. Then one can easily verify that $\varphi_{p \circ q}=\varrho \circ \mathrm{SP}^{n}\left(\varphi_{q}\right) \circ \varphi_{p}: X \rightarrow \mathrm{SP}^{m n} Z$. Thus $\varphi_{p \circ q}$ is continuous.

The cohomology transfer behaves well with respect to composite ramified covering maps.

Proposition 1.15. The following holds:

$$
t^{p \circ q}=t^{p} \circ t^{q}: H^{k}(Z ; L) \stackrel{t^{q}}{\rightarrow} H^{k}(Y ; L) \stackrel{t^{p}}{\rightarrow} H^{k}(X ; L) .
$$

Proof. We prove that $t^{p \circ q}=t^{p} \circ t^{q}:[Z, \mathcal{F}] \rightarrow[X, \mathcal{F}]$ for any topological abelian group $\mathcal{F}$. Take $w=[h] \in[Z, \mathcal{F}], v=[g] \in[Y, \mathcal{F}], u=$ $[f] \in[Z, \mathcal{F}]$. Then $v=t^{q}(w)$ if $g(y)=\sum_{q(z)=y} \nu(z) h(z)$, and $u=t^{p}(v)$ if $f(x)=\sum_{p(y)=x} \mu(y) g(y)$. Hence,

$$
f(x)=\sum_{p(y)=x} \mu(y) \sum_{q(z)=y} \nu(z) h(z)=\sum_{p q(z)=x} \mu(q(z)) \nu(z) h(z)=\sum_{p q(z)=x} \xi(z) h(z) .
$$

Therefore, $t^{q} t^{p}(u)=t^{p \circ q}(u)$.

COROLlary 1.16. Given an n-fold ramified covering map $p: E \rightarrow X$ with multiplicity function $\mu$ and an integer $l$, there is an ln-fold ramified covering map $p_{l}: E \rightarrow X$ such that $p_{l}=p$ and $\mu_{l}(e)=l \mu(e), e \in E$. Then $t^{p_{l}}=l t^{p}: H^{k}(E ; L) \rightarrow H^{k}(X ; L)$.

Proof. Consider the $l$-fold ramified covering map $q: E \rightarrow E$ such that $q=\operatorname{id}_{E}$ and $\nu(e)=l$ for all $e \in E$. Then $p_{l}=p \circ q$. Then apply Proposition 1.15 .

REMARK 1.17. The $l n$-fold covering map $p_{l}$ obtained from $l$ is a sort of spurious ramified covering map, since the multiplicity of $p$ is artificially multiplied by $l$. It is interesting to remark that the previous result shows that the transfer of this new ramified covering map $p_{l}$ remains essentially unchanged. 
2. General transfers for $n$-fold ramified covering maps in representable cofunctors. In this section we consider representable contravariant functors $h$ and $k$, that is, $h(-)=[-, \mathcal{H}]$ and $k(-)=[-, \mathcal{K}]$, where $\mathcal{H}$ and $\mathcal{K}$ are spaces, in order to study general transfers.

DEFINITION 2.1. An $(h, k)$-transfer for $n$-fold ramified covering maps $\tau$ associates, to every $n$-fold ramified covering map $p: E \rightarrow X$ with multiplicity function $\mu: E \rightarrow \mathbb{N}$, a function $\tau^{p}: h(E) \rightarrow k(X)$ with the following two properties:

(i) Given a pullback diagram

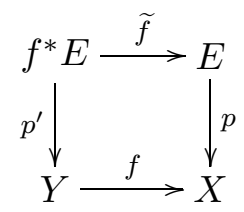

of $n$-fold ramified covering maps, the diagram

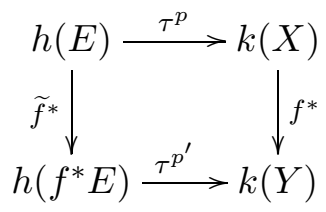

commutes.

(ii) Given $f: B \rightarrow C$, then for the diagram (1.10) the following diagram commutes:

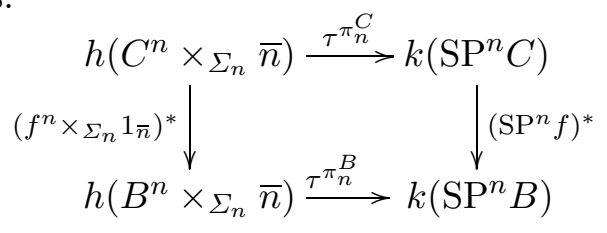

REMARK 2.3. Observe that the transfers just defined need not be homomorphisms (even when $\mathcal{H}$ and $\mathcal{K}$ are $\mathrm{H}$-spaces).

Note 2.4. Considering the category $\mathcal{R a m c o v}_{n}$ whose objects are $n$-fold ramified covering maps and whose morphisms are pullback diagrams, one has two functors, namely $\mathcal{E}, \mathcal{X}: \mathcal{R a m c o v}_{n} \rightarrow \mathcal{T}$ op such that given a covering map $p: E \rightarrow X, \mathcal{E}(p)=E$ and $\mathcal{X}(p)=X$. Then a transfer is a natural transformation $h \circ \mathcal{E} \rightarrow k \circ X$ (between functors $\mathcal{R a m c o v}_{n} \rightarrow$ Set), that also is a natural transformation $h \circ(-)^{n} \times \Sigma_{n} \bar{n} \stackrel{\dot{\rightarrow}}{\rightarrow} \circ \mathrm{SP}^{n}$ (between functors $\mathcal{T}$ op $\rightarrow$ Set).

If $h=k=H^{q}(-; L)$, then by 1.8 and 1.9 , we have the following.

Proposition 2.5. The transfer $t^{p}: h(E) \rightarrow h(X)$ defined in 1.4 is an $(h, h)$-transfer. 
We have the following classification result.

TheOREM 2.6 .

(i) Each class $w \in k\left(\mathrm{SP}^{n} \mathcal{H}\right)$ determines an $(h, k)$-transfer $\tau_{w}$ for $n$-fold ramified covering maps, and conversely

(ii) each $(h, k)$-transfer $\tau$ for $n$-fold ramified covering maps determines a class $w_{\tau} \in k\left(\mathrm{SP}^{n} \mathcal{H}\right)$; moreover,

(iii) the class associated to $\tau_{w}$ is $w$, and conversely

(iv) the transfer associated to $w_{\tau}$ is $\tau$.

Proof. (i) Take a class $w \in k\left(\mathrm{SP}^{n} \mathcal{H}\right)$ and let $p: E \rightarrow X$ be an $n$-fold ramified covering map with multiplicity function $\mu: E \rightarrow \mathbb{N}$. We define $\tau_{w}^{p}: h(E) \rightarrow k(X)$ as follows. Given $[\beta] \in h(E)$, let $\tau_{w}^{p}[\beta]$ be the homotopy class of the composite

$$
X \stackrel{\varphi_{p}}{\rightarrow} \mathrm{SP}^{n} E \stackrel{\mathrm{SP}^{n} \beta}{\longrightarrow} \mathrm{SP}^{n} \stackrel{w}{\rightarrow} \mathcal{K} .
$$

In order to show that $\tau_{w}$ has property $2.1(\mathrm{i})$, consider the pullback diagram (2.2). The element $k(f) \circ \tau_{w}^{p}[\beta]$ is given by the homotopy class of the composite

$$
Y \stackrel{f}{\rightarrow} X \stackrel{\varphi_{p}}{\rightarrow} \mathrm{SP}^{n} E \stackrel{\mathrm{SP}^{n} \beta}{\longrightarrow} \mathrm{SP}^{n} \mathcal{H} \stackrel{w}{\rightarrow} \mathcal{K}
$$

On the other hand, the element $\tau_{w}^{p^{\prime}} \circ h(\tilde{f})[\beta]$ is given by the homotopy class of the composite

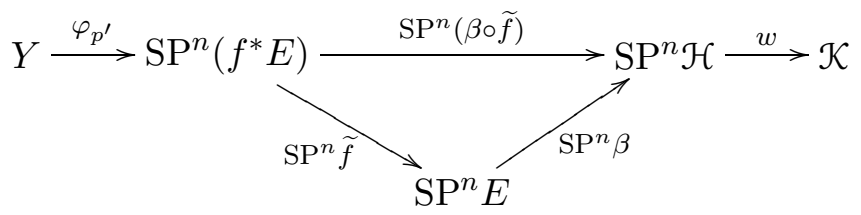

By the functoriality of the construction $\mathrm{SP}^{n}$, the triangle commutes; therefore, we only have to show the commutativity of the diagram

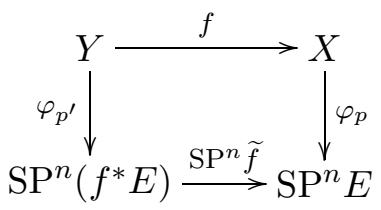

To that end, take $y \in Y$ and consider the fiber $p^{-1}(f(y))=\left\{e_{1}, \ldots, e_{r}\right\}$. Then

$$
\varphi_{p} f(y)=\langle\underbrace{e_{1}, \ldots, e_{1}}_{\mu\left(e_{1}\right)}, \ldots, \underbrace{e_{r}, \ldots, e_{r}}_{\mu\left(e_{r}\right)}\rangle .
$$

Since $p^{\prime-1}(y)=\left\{\left(y, e_{1}\right), \ldots,\left(y, e_{r}\right)\right\}$ and the multiplicity function of $p^{\prime}$ is 
$\mu \circ \widetilde{f}$, we have

$$
\varphi_{p^{\prime}}(y)=\langle\underbrace{\left(y, e_{1}\right), \ldots,\left(y, e_{1}\right)}_{\mu\left(e_{1}\right)}, \ldots, \underbrace{\left(y, e_{r}\right), \ldots,\left(y, e_{r}\right)}_{\mu\left(e_{r}\right)}\rangle .
$$

Therefore,

$$
\operatorname{SP}^{n} \tilde{f} \varphi_{p^{\prime}}(y)=\langle\underbrace{e_{1}, \ldots, e_{1}}_{\mu\left(e_{1}\right)}, \ldots, \underbrace{e_{r}, \ldots, e_{r}}_{\mu\left(e_{r}\right)}\rangle,
$$

and so the diagram commutes.

It is immediate to see that $\tau_{w}$ has property 2.1 (ii), by the functoriality of $\mathrm{SP}^{n}$ and since $\mathrm{SP}^{n}\left(f^{n} \times \Sigma_{n} \mathrm{id}_{\bar{n}}\right) \circ \varphi_{\pi_{n}^{C}}=\varphi_{\pi_{n}^{B}} \circ \mathrm{SP}^{n} f$, as one easily verifies.

(ii) Let $\tau$ be an $(h, k)$-transfer for $n$-fold ramified covering maps and consider the map $\pi: \mathcal{H}^{n} \times{ }_{\Sigma_{n}} \bar{n} \rightarrow \mathrm{SP}^{n} \mathcal{H}$. As remarked in 1.3, $\pi$ is an $n$-fold ramified covering map. Therefore, we have $\tau^{\pi}: h\left(\mathcal{H}^{n} \times_{\Sigma_{n}} \bar{n}\right) \rightarrow k\left(\mathrm{SP}^{n} \mathcal{H}\right)$. Let $\alpha: \mathcal{H}^{n} \times \Sigma_{n} \bar{n} \rightarrow \mathcal{H}$ be given by $\alpha\left\langle a_{1}, \ldots, a_{n} ; i\right\rangle=a_{i}$. Then $[\alpha] \in$ $h\left(\mathcal{H}^{n} \times{ }_{\Sigma_{n}} \bar{n}\right)$. We associate to $\tau$ the element $w_{\tau}=\tau^{\pi}[\alpha] \in k\left(\mathrm{SP}^{n} \mathcal{H}\right)$.

(iii) Let $w \in k\left(\mathrm{SP}^{n} \mathcal{H}\right)$, and consider the associated transfer $\tau_{w}$. The class in $k\left(\mathrm{SP}^{n} \mathcal{H}\right)$ determined by $\tau_{w}$ is given by $\tau_{w}^{\pi}[\alpha]$, where $\alpha: \mathcal{H}^{n} \times_{\Sigma_{n}} \bar{n} \rightarrow \mathcal{H}$ is given by $\alpha\left\langle a_{1}, \ldots, a_{n} ; i\right\rangle=a_{i}$. Therefore, $\tau_{w}^{\pi}[\alpha]$ is the homotopy class of the composite

$$
\mathrm{SP}^{n} \mathcal{H} \stackrel{\varphi_{\pi}}{\longrightarrow} \mathrm{SP}^{n}\left(\mathcal{H}^{n} \times_{\Sigma_{n}} \bar{n}\right) \stackrel{\mathrm{SP}^{n} \alpha}{\longrightarrow} \mathrm{SP}^{n} \mathcal{H} \stackrel{w}{\rightarrow} \mathcal{K}
$$

Let $a=\left\langle a_{1}, \ldots, a_{n}\right\rangle$ be an element in $\mathrm{SP}^{n} \mathcal{H}$. Then

$$
\varphi_{\pi}(a)=\left\langle\left\langle a_{1}, \ldots, a_{n} ; 1\right\rangle, \ldots,\left\langle a_{1}, \ldots, a_{n} ; n\right\rangle\right\rangle .
$$

Therefore,

$$
\mathrm{SP}^{n} \alpha \circ \varphi_{\pi}(a)=\left\langle a_{1}, \ldots, a_{n}\right\rangle=a,
$$

so that $\mathrm{SP}^{n} \alpha \circ \varphi_{\pi}=1$. Hence $\tau_{w}^{\pi}[\alpha]=w$.

(iv) Finally, given an $(h, k)$-transfer $\tau$, we have $w_{\tau}=\tau^{\pi}[\alpha]$. In order to show that $\tau_{w_{\tau}}=\tau$, consider an $n$-fold ramified covering map $p: E \rightarrow X$ with multiplicity function $\mu: E \rightarrow \mathbb{N}$ and some element $[\beta] \in h(E)=[E, \mathcal{H}]$. We shall prove that $\tau_{w_{\tau}}^{p}[\beta]=\tau^{p}[\beta]$. For that, consider the following two diagrams:
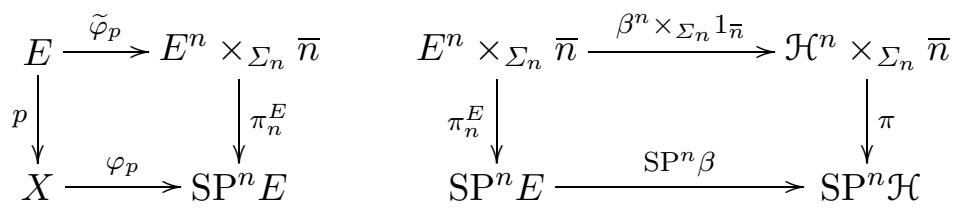

The left one is a pullback diagram while the one on the right is like (1.10). Hence, by the two properties of the transfer, we have two commutative 
diagrams

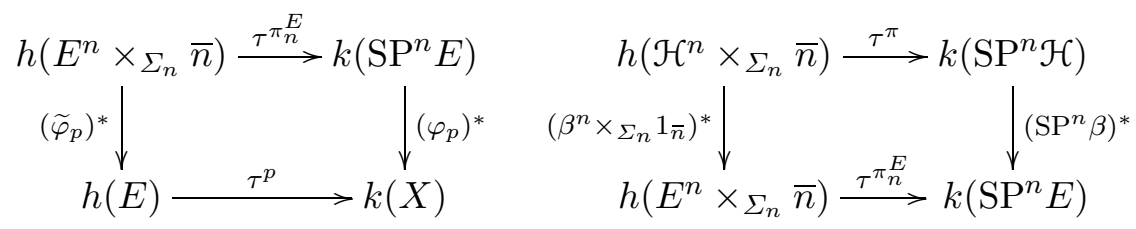

and putting the right one on top of the left one, we obtain

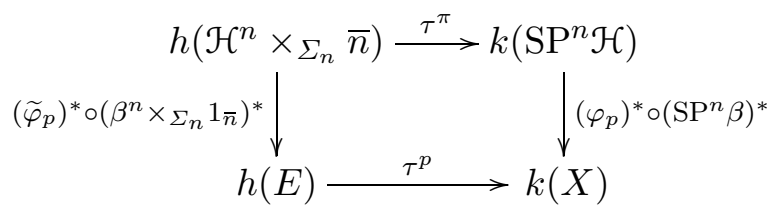

If we now chase our element $[\alpha] \in h\left(\mathcal{H}^{n} \times_{\Sigma_{n}} \bar{n}\right)$ defined in the proof of (ii) along the top and right side of the diagram, we obtain $\left[w_{\tau} \circ \operatorname{SP}^{n} \beta \circ \varphi_{p}\right]=$ $\tau_{w_{\tau}}^{p}[\beta]$, while if we chase it along the left and bottom side of the diagram we obtain $\tau^{p}[\beta]$. Thus $\tau_{w_{\tau}}^{p}[\beta]=\tau^{p}[\beta]$, as desired.

As a consequence of Theorem 2.6, we obtain the following.

Corollary 2.7. There is a one-to-one correspondence between $(h, k)$ transfers $\tau$ and elements $w$ in $k\left(\mathrm{SP}^{n} \mathcal{H}\right)$.

In the following result we compute $w_{t}$ for the cohomology transfers $t^{p}$, $p: E \rightarrow X$, defined in 1.4 .

Proposition 2.8. Let $\mathcal{H}=F\left(\mathbb{S}^{q}, L\right)$. Then the element $w_{t} \in\left[\mathrm{SP}^{n} \mathcal{H}, \mathcal{H}\right]$ that corresponds to the transfer $t^{p}$ is given by

$$
w_{t}\left\langle a_{1}, \ldots, a_{n}\right\rangle=a_{1}+\cdots+a_{n} .
$$

Proof. Let $\pi: \mathcal{H}^{n} \times_{\Sigma_{n}} \bar{n} \rightarrow \mathrm{SP}^{n} \mathcal{H}$ be the $n$-fold ramified covering map given above. The transfer $t^{\pi}: h\left(\mathcal{H}^{n} \times \Sigma_{n} \bar{n}\right) \rightarrow h\left(\mathrm{SP}^{n} \mathcal{H}\right)$ is such that $t^{\pi}[\alpha](x)=\sum_{\pi(e)=x} \mu(e) \alpha(e)$. Thus, if $x=\left\langle a_{1}, \ldots, a_{n}\right\rangle$, then $\pi^{-1}(x)=$ $\left\{\left\langle a_{1}, \ldots, a_{n} ; i\right\rangle \mid i=1, \ldots, n\right\}$. Hence

$$
w_{t}(x)=t^{\pi}[\alpha](x)=\sum_{i=1}^{n} \alpha\left\langle a_{1}, \ldots, a_{n} ; i\right\rangle=\sum_{i=1}^{n} a_{i} .
$$

Definition 2.9. By Theorem 2.6, given representable functors $h$ and $k$, we can define the set of transfers from $h(E)$ to $k(X)$ for each $n$-fold ramified covering map $p: E \rightarrow X$. We denote this set by $T_{n}^{R}(h, k)$. If we assume that the functor $k$ takes values in the category $\mathcal{A b}$ of abelian groups, then we can give $T_{n}^{R}(h, k)$ a group structure as follows. Given $\sigma, \tau \in T_{n}^{R}(h, k)$ and an $n$-fold ramified covering map $p: E \rightarrow X$, we define the transfer $\sigma+\tau$ by $(\sigma+\tau)^{p}(a)=\sigma^{p}(a)+\tau^{p}(a)$, for every $a \in h(E)$. 
Corollary 2.10. Assume that $k$ takes values in $\mathcal{A b}$. Then the bijection of Corollary 2.7 gives an isomorphism of abelian groups

$$
T_{n}^{R}(h, k) \cong k\left(\operatorname{SP}^{n}(\mathcal{H})\right) .
$$

Proof. By 2.7, there is a bijection $\psi: T_{n}^{R}(h, k) \rightarrow k\left(\mathrm{SP}^{n}(\mathcal{H})\right)$ given by $\psi(\tau)=w_{\tau}=\tau^{\pi}[\alpha]$, as in the proof of 2.6(ii). Then

$$
\psi(\sigma+\tau)=(\sigma+\tau)^{\pi}[\alpha]=\sigma^{\pi}[\alpha]+\tau^{\pi}[\alpha]=\psi(\sigma)+\psi(\tau) .
$$

Therefore, $\psi$ is an isomorphism.

The following is a nice consequence of this corollary.

Proposition 2.11. Let $\tau$ be an $(h, k)$-transfer and assume that there is a commutative diagram

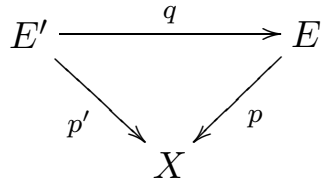

of $n$-fold ramified covering maps such that $q: E^{\prime} \rightarrow E$ is surjective and $\mu(e)=\sum_{e^{\prime} \in q^{-1}(e)} \mu^{\prime}\left(e^{\prime}\right)$. Then the following triangle commutes:

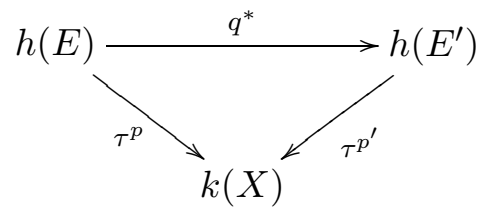

Proof. By the classification result 2.7, there is an element $w=w_{\tau} \in$ $k\left(\mathrm{SP}^{n} \mathcal{H}\right)$ such that for any $p: E \rightarrow X$ and any element $[f] \in h(E)=[E, \mathcal{H}]$, $\tau^{p}[f]$ is given by the class of the composite

$$
X \stackrel{\varphi_{p}}{\rightarrow} \mathrm{SP}^{n} E \stackrel{\mathrm{SP}^{n} f}{\longrightarrow} \mathrm{SP}^{n} \mathcal{H} \stackrel{w}{\rightarrow} \mathcal{K} .
$$

Similarly for $\tau^{p^{\prime}}\left[f^{\prime}\right], f^{\prime}=f \circ q$. Consider the following diagram:

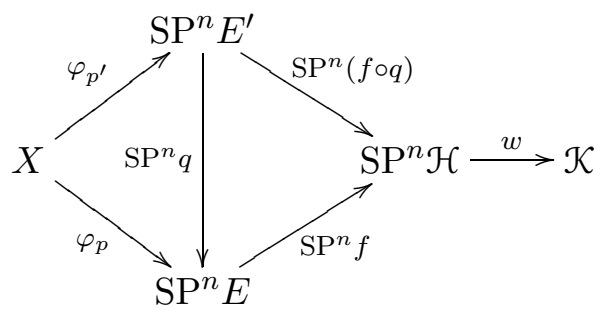

The triangle on the right clearly commutes. We shall show that so does the left one. If $p^{-1}(x)=\left\{e_{1}, \ldots, e_{r}\right\}$ and $p^{\prime-1}(x)=\left\{e_{1}^{\prime}, \ldots, e_{s}^{\prime}\right\}$, then

$$
\varphi_{p}(x)=\langle\underbrace{e_{1}, \ldots, e_{1}}_{\mu\left(e_{1}\right)}, \ldots, \underbrace{e_{r}, \ldots, e_{r}}_{\mu\left(e_{r}\right)}\rangle, \quad \varphi_{p^{\prime}}(x)=\langle\underbrace{e_{1}^{\prime}, \ldots, e_{1}^{\prime}}_{\mu^{\prime}\left(e_{1}^{\prime}\right)}, \ldots, \underbrace{e_{s}^{\prime}, \ldots, e_{s}^{\prime}}_{\mu^{\prime}\left(e_{s}^{\prime}\right)}\rangle .
$$


Observe that $p^{\prime-1}(x)=q^{-1}\left(e_{1}\right) \sqcup \cdots \sqcup q^{-1}\left(e_{r}\right)$. Rewrite

$$
\varphi_{p^{\prime}}(x)=\langle\underbrace{e_{11}^{\prime}, \ldots, e_{11}^{\prime}}_{\mu^{\prime}\left(e_{11}^{\prime}\right)}, \ldots, \underbrace{e_{1 s_{1}}^{\prime}, \ldots, e_{1 s_{1}}^{\prime}}_{\mu^{\prime}\left(e_{1 s_{1}}^{\prime}\right)}, \ldots, \underbrace{e_{r 1}^{\prime}, \ldots, e_{r 1}^{\prime}}_{\mu^{\prime}\left(e_{r 1}^{\prime}\right)}, \ldots, \underbrace{e_{r s_{r}}^{\prime}, \ldots, e_{r s_{r}}^{\prime}}_{\mu^{\prime}\left(e_{r s_{r}}^{\prime}\right)}\rangle,
$$

where $q^{-1}\left(e_{1}\right)=\left\{e_{11}^{\prime}, \ldots, e_{1 s_{1}}^{\prime}\right\}, \ldots, q^{-1}\left(e_{r}\right)=\left\{e_{r 1}^{\prime}, \ldots, e_{r s_{r}}^{\prime}\right\}$. Since $\mu\left(e_{1}\right)=$ $\mu^{\prime}\left(e_{11}^{\prime}\right)+\cdots+\mu^{\prime}\left(e_{1 s_{1}}^{\prime}\right), \ldots, \mu\left(e_{r}\right)=\mu^{\prime}\left(e_{r 1}^{\prime}\right)+\cdots+\mu^{\prime}\left(e_{r s_{r}}^{\prime}\right)$, we have

$$
\begin{aligned}
& \mathrm{SP}^{n} q \varphi_{p^{\prime}}(x) \\
& =\mathrm{SP}^{n} q\langle\underbrace{e_{11}^{\prime}, \ldots, e_{11}^{\prime}}_{\mu^{\prime}\left(e_{11}^{\prime}\right)}, \ldots, \underbrace{e_{1 s_{1}}^{\prime}, \ldots, e_{1 s_{1}}^{\prime}}_{\mu^{\prime}\left(e_{1 s_{1}}^{\prime}\right)}, \ldots, \underbrace{e_{r 1}^{\prime}, \ldots, e_{r 1}^{\prime}}_{\mu^{\prime}\left(e_{r 1}^{\prime}\right)}, \ldots, \underbrace{e_{r s_{r}}^{\prime}, \ldots, e_{r s_{r}}^{\prime}}_{\mu^{\prime}\left(e_{r s_{r}}^{\prime}\right)}\rangle \\
& =\langle\underbrace{e_{1}, \ldots, e_{1} \ldots, e_{1}, \ldots, e_{1}}_{\mu\left(e_{1}\right)}, \ldots, \underbrace{e_{r}, \ldots, e_{r} \ldots, e_{r}, \ldots, e_{r}}_{\mu\left(e_{r}\right)}\rangle=\varphi_{p}(x) .
\end{aligned}
$$

Hence,

$$
\tau^{p}[f]=\tau_{w}^{p}[f]=\tau_{w}^{p^{\prime}}[f \circ q]=\tau^{p^{\prime}} q^{*}[f] .
$$

The following theorem yields the existence of transfers in some cases.

THEOREM 2.13. Let $H^{*}$ denote singular cohomology with coefficients in $\mathbb{Z}$. Then

$$
T_{n}^{R}\left(H^{r}, H^{s}\right) \cong \begin{cases}0 & \text { if } n \geq s<r(s>0), \\ \mathbb{Z} & \text { if } n \geq s=r \\ 0 & \text { if } n \geq s=r+1 .\end{cases}
$$

Proof. By 2.6 and 2.7, we have an isomorphism

$$
T_{n}^{R}\left(H^{r}, H^{s}\right) \cong H^{s}\left(\mathrm{SP}^{n}(K(\mathbb{Z}, r)) .\right.
$$

By $[2,6.3 .24]$, for any $(r-1)$-connected CW-complex $X$, the inclusion $X \hookrightarrow$ $\mathrm{SP}^{\infty} X$ is an $(r+1)$-equivalence. Therefore, $\mathrm{SP}^{\infty} K(\mathbb{Z}, r)$ is $(r-1)$-connected, $\pi_{r}\left(\mathrm{SP}^{\infty} K(\mathbb{Z}, r)\right) \cong \mathbb{Z}$, and $\pi_{r+1}\left(\mathrm{SP}^{\infty} K(\mathbb{Z}, r)\right)=0$. By the Hurewicz theorem,

$$
\begin{aligned}
& \widetilde{H}_{i}\left(\mathrm{SP}^{\infty} K(\mathbb{Z}, r)\right)=0 \quad \text { for } i<r \\
& H_{r}\left(\mathrm{SP}^{\infty} K(\mathbb{Z}, r)\right) \cong \mathbb{Z}, \quad H_{r+1}\left(\operatorname{SP}^{\infty} K(\mathbb{Z}, r)\right)=0 .
\end{aligned}
$$

By the universal coefficients theorem,

$$
\begin{gathered}
H^{s}\left(\mathrm{SP}^{\infty} K(\mathbb{Z}, r)\right)=0 \quad \text { for } s<r, \\
H^{r}\left(\mathrm{SP}^{\infty} K(\mathbb{Z}, r)\right) \cong \operatorname{Hom}\left(H_{r}\left(\mathrm{SP}^{\infty} K(\mathbb{Z}, r)\right) ; \mathbb{Z}\right) \cong \operatorname{Hom}(\mathbb{Z}, \mathbb{Z}) \cong \mathbb{Z} .
\end{gathered}
$$

Since $H_{r}\left(\mathrm{SP}^{\infty} K(\mathbb{Z}, r)\right) \cong \mathbb{Z}$, it follows that $\operatorname{Ext}\left(H_{r}\left(\mathrm{SP}^{\infty} K(\mathbb{Z}, r)\right) ; \mathbb{Z}\right)=0$, and we have $H^{r+1}\left(\mathrm{SP}^{\infty} K(\mathbb{Z}, r)\right) \cong \operatorname{Hom}\left(H_{r}\left(\mathrm{SP}^{\infty} K(\mathbb{Z}, r)\right) ; \mathbb{Z}\right)=0$. By [8], for any $\mathrm{CW}$-complex $X, H^{s}\left(\mathrm{SP}^{\infty} X\right) \cong H^{s}\left(\mathrm{SP}^{n} X\right)$ for $n \geq s$, so the result follows. 
3. Comparison between transfers for ordinary covering maps and for ramified covering maps. In this section we shall compare our classification of transfers for $n$-fold ramified covering maps given in the previous section with the classification of transfers for $n$-fold ordinary covering maps obtained by Roush [6]. For a description of his result we follow [1].

Definition 3.1. Take again $h(-)=[-, \mathcal{H}]$ and $k(-)=[-, \mathcal{K}]$ as above. An $(h, k)$-transfer for $n$-fold covering maps associates to every $n$-fold covering map $p: E \rightarrow X$ over a paracompact space $X$ a function $t^{p}$ : $h(E) \rightarrow k(X)$, which is natural with respect to pullbacks in the sense of property $(2.1)(\mathrm{i})$ in the definition of $(h, k)$-transfers for $n$-fold ramified covering maps.

Denote by $T_{n}(h, k)$ the set of transfers for $n$-fold ordinary covering maps, and let $E \Sigma_{n} \rightarrow B \Sigma_{n}$ be the universal principal $\Sigma_{n}$-bundle. Then we have the following.

THEOREM 3.2 (Roush). There is a bijection

$$
\left[E \Sigma_{n} \times \Sigma_{n} \mathcal{H}^{n}, \mathcal{K}\right] \rightarrow T_{n}(h, k) .
$$

Since the transfers for $n$-fold ramified covering maps are also natural with respect to pullbacks, as just mentioned above, we have a restriction function $r: T_{n}^{R}(h, k) \rightarrow T_{n}(h, k)$. The following theorem relates both classifications, namely Theorems 3.2 and 2.7 .

Theorem 3.3. Let $\varrho: E \Sigma_{n} \times{ }_{\Sigma_{n}} \mathcal{H}^{n} \rightarrow \mathrm{SP}^{n} \mathcal{H}$ be given by $\varrho\left\langle e ; a_{1}, \ldots, a_{n}\right\rangle$ $=\left\langle a_{1}, \ldots, a_{n}\right\rangle$. Then the following diagram commutes:

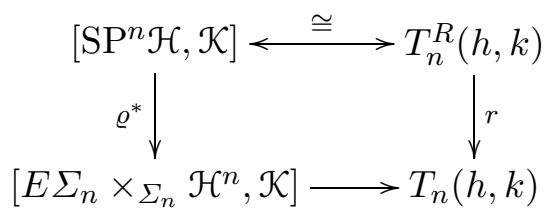

Proof. Let $w: \mathrm{SP}^{n} \mathcal{H} \rightarrow \mathcal{K}$ be a map and $\tau_{w}$ the transfer for $n$-fold ramified covering maps associated to it according to Corollary 2.7. Consider $\varrho^{*}[w]=[w \circ \varrho]$ and let $p: E \rightarrow X$ be an $n$-fold covering map and $g: E \rightarrow \mathcal{H}$. The value at $[g]$ of the transfer $\tau^{\prime p}$ associated to the class $\varrho^{*}[w]$ is defined as follows. Let $q: \bar{E} \rightarrow X$ be the principal $\Sigma_{n^{-}}$ bundle associated to $p$, i.e., $\bar{E}=\left\{\left(e_{1}, \ldots, e_{n}\right) \in E^{n} \mid e_{i} \neq e_{j}\right.$ if $i \neq j$, and $\left.p\left(e_{1}\right)=\cdots=p\left(e_{n}\right)\right\}$, and $q\left(e_{1}, \ldots, e_{n}\right)=p\left(e_{1}\right)$. There is a free $\Sigma_{n^{-}}$ action on $\bar{E}$ defined by permuting coordinates, and a homeomorphism $\gamma: \bar{E} / \Sigma_{n} \rightarrow X$ given by $\gamma\left\langle e_{1}, \ldots e_{n}\right\rangle=p\left(e_{1}\right)$. Therefore there is a pull- 
back square

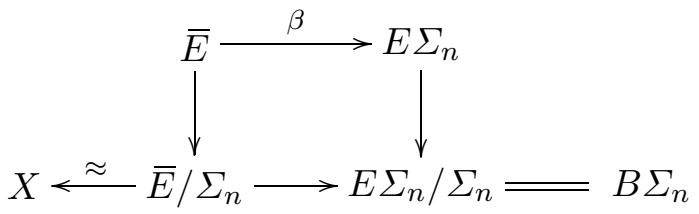

Then $\tau^{\prime p}[g] \in k(X)=[X, \mathcal{K}]$ is the class of the composite

$$
X \approx \bar{E} / \Sigma_{n} \stackrel{\psi}{\longrightarrow} E \Sigma_{n} \times_{\sigma_{n}} E^{n} \stackrel{\text { id } \times_{\Sigma_{n}} g^{n}}{\longrightarrow} E \Sigma_{n} \times \Sigma_{n} \mathcal{H}^{n} \stackrel{w \circ \varrho}{\longrightarrow} \mathcal{K}
$$

where $\psi\left\langle e_{1}, \ldots, e_{n}\right\rangle=\left\langle\beta\left(e_{1}, \ldots, e_{n}\right) ; e_{1}, \ldots, e_{n}\right\rangle$. Now we consider the following diagram:

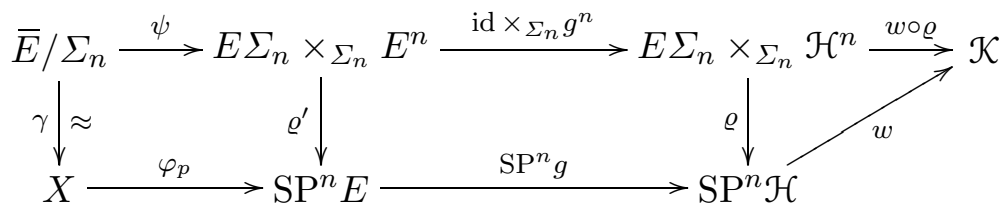

where $\varrho^{\prime}\left\langle a ; e_{1}, \ldots, e_{n}\right\rangle=\left\langle e_{1}, \ldots, e_{n}\right\rangle$. Since $p: E \rightarrow X$ is an $n$-fold covering map, $\mu(e)=1$ for all $e \in E$, and $\left\{e_{1}, \ldots, e_{n}\right\}$ is the fiber over $p\left(e_{1}\right)$. Therefore, $\varphi_{p}\left(\gamma\left\langle e_{1}, \ldots, e_{n}\right\rangle\right)=\left\langle e_{1}, \ldots, e_{n}\right\rangle$. Since $\varrho^{\prime} \psi\left\langle e_{1}, \ldots, e_{n}\right\rangle=\left\langle e_{1}, \ldots, e_{n}\right\rangle$, the left square of the diagram commutes, and the middle square as well as the triangle are clearly also commutative. But the class of the composite $w \circ \mathrm{SP}^{n} g \circ \varphi_{p}$ is $r\left(\tau_{w}\right)^{p}[g]=\tau_{w}^{p}[g]$. Hence $r\left(\tau_{w}\right)^{p}[g]=\tau^{\prime p}[g]$ and thus $r\left(\tau_{w}\right)=\tau^{\prime}$.

\section{Transfers for ramified covering maps in representable cofunc-}

tors. In this section we shall consider families of $(h, k)$-transfers for $n$-fold ramified covering maps for all $n$, and in order to do this, we assume that $\mathcal{H}$ has a base point $*$.

Before stating the relevant definition, consider an $n$-fold ramified covering map $p: E \rightarrow X$ with multiplicity function $\mu: E \rightarrow \mathbb{N}$. Recall the $(n+1)$-fold ramified covering map $\bar{p}: \bar{E}=E \sqcup X \rightarrow X$ given in Remark 1.2.

Definition 4.1. An $(h, k)$-transfer $\tau$ for ramified covering maps consists of an $(h, k)$-transfer $\tau_{n}$ for $n$-fold ramified covering maps, for each $n \in \mathbb{N}$, together with the following stability condition. Given an $n$-fold ramified covering map $p: E \rightarrow X$, the following diagram commutes:

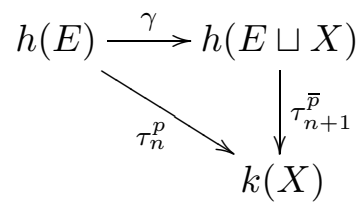

where $\gamma[f]=[(f, \kappa)], \kappa$ constant on $X$ with value the base point in $\mathcal{H}$. 
Proposition 4.3. Define $\iota: T_{n+1}^{R}(h, k) \rightarrow T_{n}^{R}(h, k)$ by $\tau_{n+1} \mapsto \widehat{\tau}_{n}$, where for an $n$-fold ramified covering map $p: E \rightarrow X, \widehat{\tau}_{n}^{p}=\tau_{n+1}^{\bar{p}} \circ \gamma$. Then the diagram

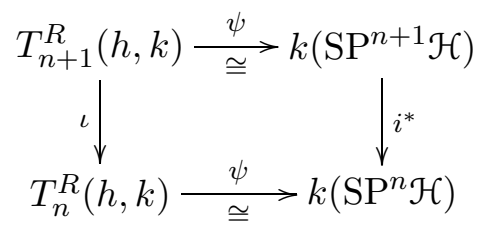

commutes for all $n$, where $i: \mathrm{SP}^{n} \mathcal{H} \hookrightarrow \mathrm{SP}^{n+1} \mathcal{H}$ is the canonical inclusion given by $\left\langle a_{1}, \ldots, a_{n}\right\rangle \mapsto\left\langle a_{1}, \ldots, a_{n}, *\right\rangle$.

Proof. Let $p: E \rightarrow \mathrm{SP}^{n} \mathcal{H}$ be the $(n+1)$-fold ramified covering map obtained by taking the pullback of $\pi_{n+1}$ over $i: \mathrm{SP}^{n} \mathcal{H} \hookrightarrow \mathrm{SP}^{n+1} \mathcal{H}$. Consider the maps $\beta: \mathcal{H}^{n} \times_{\Sigma_{n}} \bar{n} \sqcup \mathrm{SP}^{n} \mathcal{H} \rightarrow \mathcal{H}^{n+1} \times_{\Sigma_{n+1}} \overline{n+1}$ given by $\beta\left\langle a_{1}, \ldots, a_{n} ; j\right\rangle=\left\langle a_{1}, \ldots, a_{n}, * ; j\right\rangle$ and $\beta\left\langle a_{1}, \ldots, a_{n}\right\rangle=\left\langle a_{1}, \ldots, a_{n}, * ; n+1\right\rangle$, and $\pi_{n}: \mathcal{H}^{n} \times_{\Sigma_{n}} \bar{n} \rightarrow \mathrm{SP}^{n} \mathcal{H}$, the canonical surjection. Hence we have a commutative square

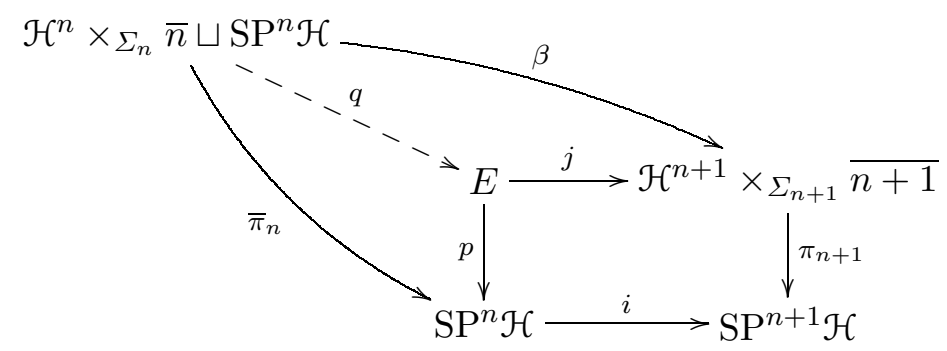

and since $E$ is a pullback, there is a (unique) map $q:\left(\mathcal{H}^{n} \times{ }_{\Sigma_{n}} \bar{n}\right) \sqcup \mathrm{SP}^{n} \mathcal{H} \rightarrow E$ such that $p \circ q=\bar{\pi}_{n}$ and $j \circ q=\beta$. We shall write an element $\langle a\rangle \in \operatorname{SP}^{n} \mathcal{H}$ as

$$
\langle a\rangle=\langle\underbrace{a_{1}, \ldots, a_{1}}_{i_{1}}, \ldots, \underbrace{a_{r}, \ldots, a_{r}}_{i_{r}}\rangle .
$$

Then $\bar{\pi}_{n}^{-1}\langle a\rangle=\left\{\left\langle a ; i_{1}\right\rangle,\left\langle a ; i_{1}+i_{2}\right\rangle, \ldots,\langle a ; n\rangle,\langle a\rangle\right\}$. On the other hand, $p^{-1}\langle a\rangle=\{\langle a\rangle\} \times\left\{\left\langle a, * ; i_{1}\right\rangle,\left\langle a, * ; i_{1}+i_{2}\right\rangle, \ldots,\langle a, * ; n\rangle,\langle a, * ; n+1\rangle\right\}$. We distinguish two cases, namely, when $a_{i} \neq *$ for all $i$, and when $a_{i}=*$ for some $i$. In the first case, $q$ induces a bijection between both fibers and one easily sees that $\mu_{p} \circ q=\mu_{\bar{\pi}_{n}}$. In the second case, arrange the components of $a$ so that

$$
\langle a\rangle=\langle\underbrace{a_{1}, \ldots, a_{1}}_{i_{1}}, \ldots, \underbrace{*, \ldots, *}_{i_{r}}\rangle .
$$

In this case, $q$ is as in the first case, except that $q\langle a ; n\rangle=q\langle a\rangle=\langle a, * ; n\rangle=$ $\langle a, * ; n+1\rangle$, so that $q$ induces a surjection between the fibers. Since $\bar{\pi}_{n}$ is also surjective, $q$ is surjective. 
Now

$$
\begin{aligned}
& \mu_{p} q\left\langle a ; i_{1}\right\rangle=i_{1}=\mu_{\bar{\pi}_{n}}\left\langle a ; i_{1}\right\rangle, \ldots, \\
& \mu_{p} q\left\langle a ; i_{1}+\cdots+i_{r-1}\right\rangle=i_{r-1}=\mu_{\bar{\pi}_{n}}\left\langle a ; i_{1}+\cdots+i_{r-1}\right\rangle, \\
& \mu_{p}(\langle a\rangle,\langle a, * ; n+1\rangle)=i_{r}+1=\mu_{\bar{\pi}_{n}}\langle a ; n\rangle+\mu_{\bar{\pi}_{n}}\langle a\rangle .
\end{aligned}
$$

Hence, $\mu_{p}(e)=\sum_{e^{\prime} \in q^{-1}(e)} \mu_{\bar{\pi}_{n}}\left(e^{\prime}\right)$ for any $e \in E$. Thus, by Proposition 2.11,

$$
\tau_{n+1}^{\bar{\pi}_{n}} \circ q^{*}=\tau_{n+1}^{p} .
$$

Let $\alpha_{n+1}: \mathcal{H}^{n+1} \times_{\Sigma_{n+1}} \overline{n+1} \rightarrow \mathcal{H}$ be given by $\alpha_{n+1}\left\langle a_{1}, \ldots, a_{n+1} ; j\right\rangle=a_{j}$, as before, and take $\tau_{n+1} \in T_{n+1}^{R}(h, k)$. Then $\beta^{*}\left(\alpha_{n+1}\right)=\gamma\left(\alpha_{n}\right)$. By the pullback property, the commutativity of the diagram, and (4.4) we have

$$
\begin{aligned}
i^{*} \psi\left(\tau_{n+1}\right) & =i^{*} \tau_{n+1}^{\pi_{n+1}}\left[\alpha_{n+1}\right]=\tau_{n+1}^{p} j^{*}\left[\alpha_{n+1}\right]=\tau_{n+1}^{\bar{\pi}_{n}} q^{*} j^{*}\left[\alpha_{n+1}\right] \\
& =\tau_{n+1}^{\bar{\pi}_{n}} \beta^{*}\left[\alpha_{n+1}\right]=\tau_{n+1}^{\bar{\pi}_{n}} \gamma\left[\alpha_{n}\right]=\widehat{\tau}_{n}^{\pi_{n}}\left[\alpha_{n}\right]=\psi\left(\widehat{\tau}_{n}\right)=\psi \iota\left(\tau_{n+1}\right)
\end{aligned}
$$

We have an inverse system

$$
\cdots \rightarrow T_{n+1}^{R}(h, k) \stackrel{\iota}{\rightarrow} T_{n}^{R}(h, k) \rightarrow \cdots \rightarrow T_{1}^{R}(h, k)=\operatorname{Nat}(h, k),
$$

where $\operatorname{Nat}(h, k)$ denotes the natural transformations from $h$ to $k$. Thus, by the previous proposition, a transfer for ramified covering maps is an element in $\lim _{n} T_{n}^{R}(h, k)=T_{\infty}^{R}(h, k)$. On the other hand, we have another inverse system

$$
\cdots \rightarrow k\left(\mathrm{SP}^{n+1} \mathcal{H}\right) \stackrel{i^{*}}{\rightarrow} k\left(\mathrm{SP}^{n} \mathcal{H}\right) \rightarrow \cdots \rightarrow k(\mathcal{H})
$$

By the commutative diagram of Proposition 4.3 and Corollary 2.10, we have the following.

THEOREM 4.5. There is an isomorphism $T_{\infty}^{R}(h, k) \rightarrow \lim _{n} k\left(\mathrm{SP}^{n} \mathcal{H}\right)$.

ExAmple 4.6. The $(h, h)$-transfers $\tau_{n}^{p}=t^{p}$ given in Definition 1.4 for each $n$ determine an $(h, h)$-transfer for ramified covering maps, since for any $[\widetilde{\alpha}] \in h(E)$ we have $\gamma[\widetilde{\alpha}]=\left[\widetilde{\alpha}^{\prime}\right]$, where $\left.\widetilde{\alpha}^{\prime}\right|_{E}=\widetilde{\alpha}$, and $\left.\widetilde{\alpha}^{\prime}\right|_{X} \equiv 0 \in \mathcal{H}$. Hence $\tau_{n+1}^{\bar{p}} \gamma[\widetilde{\alpha}]=t^{\bar{p}}\left[\widetilde{\alpha}^{\prime}\right]=\left[\alpha^{\prime}\right]$, where $\alpha^{\prime}(x)=\sum_{\bar{p}(\bar{e})=x} \bar{\mu}(\bar{e}) \widetilde{\alpha}^{\prime}(\bar{e})=\sum_{p(e)=x} \mu(e) \widetilde{\alpha}(e)$, since for $e=x, \widetilde{\alpha}^{\prime}(e)=0$. Thus $\tau_{n+1}^{\bar{p}} \circ \gamma=\tau_{n}^{p}$, and hence diagram (4.2) commutes in this case.

Assume in what follows that $\tau$ is an $(h, h)$-transfer for ramified covering maps given by an element $[w] \in \lim _{n}\left[\mathrm{SP}^{n} \mathcal{H}, \mathcal{H}\right], h=[-, \mathcal{H}]$. Let $\mathcal{H}$ be a CW-complex. Then by [9], $\mathrm{SP}^{n-1} \mathcal{H}$ is a subcomplex of $\mathrm{SP}^{n} \mathcal{H}$, so that the inclusion $i_{n}$ : $\mathrm{SP}^{n-1} \mathcal{H} \hookrightarrow \mathrm{SP}^{n} \mathcal{H}$ is a cofibration and we may thus assume that $[w]$ is given by a family of maps $w_{n}: \mathrm{SP}^{n} \mathcal{H} \rightarrow \mathcal{H}$ such that $w_{n-1}=$ $w_{n} \circ i_{n}$. If we further assume that $\tau_{X}=1_{h(X)}$, then $w_{1} \simeq \mathrm{id} \mathcal{H}_{\mathcal{H}}$, and we may suppose from the start that $w_{1}=\mathrm{id} \mathcal{H}$. Thus the maps $w_{n}$ determine a map 
$w: \mathrm{SP}^{\infty} \mathcal{H} \rightarrow \mathcal{H}$ such that $\left.w\right|_{\mathrm{SP}^{n} \mathcal{H}}=w_{n}$. In particular, $\left.w\right|_{\mathcal{H}}=\mathrm{id}_{\mathcal{H}}$. We have the following.

LEMMA 4.7. Let $\mathcal{H}$ have the homotopy type of a connected $\mathrm{CW}$-complex. If there is a map $w: \mathrm{SP}^{\infty} \mathcal{H} \rightarrow \mathcal{H}$ such that $\left.w\right|_{\mathcal{H}}=\mathrm{id}_{\mathcal{H}}$, then $\mathcal{H}$ has the homotopy type of a weak product $\widetilde{\prod}_{n \geq 1} K\left(\pi_{n}(\mathcal{H}), n\right)$ of Eilenberg-Mac Lane spaces.

Proof. The homomorphism $w_{*}: \pi_{n}\left(\mathrm{SP}^{\infty} \mathcal{H}\right) \rightarrow \pi_{n}(\mathcal{H})$ splits $i_{*}: \pi_{n}(\mathcal{H}) \rightarrow$ $\pi_{n}\left(\mathrm{SP}^{\infty} \mathcal{H}\right)$ for all $n$. By the Dold-Thom theorem (see [2]), $\pi_{n}\left(\mathrm{SP}^{\infty} \mathcal{H}\right) \cong$ $\widetilde{H}_{n}(\mathcal{H} ; \mathbb{Z})$ and under this isomorphism, $i_{*}$ corresponds to the Hurewicz homomorphism. Thus, the Hurewicz homomorphism is a split mono and hence by a theorem of Moore (see [10, p. 420]) we have the result.

Hence, by the previous lemma and Theorem 4.5, we have the following consequence.

TheOREM 4.8. Let $\mathcal{H}$ have the homotopy type of a connected $\mathrm{CW}$-complex. There is an $(h, h)$-transfer $\tau$ for ramified covering maps such that $\tau_{1}^{\operatorname{id}_{X}}=1_{h(X)}$ if and only if $\mathcal{H}$ has the homotopy type of a weak product

$$
\widetilde{\prod_{n \geq 1}} K\left(\pi_{n}(\mathcal{H}), n\right)
$$

of Eilenberg-Mac Lane spaces.

In what follows, we shall show that the transfers for ramified covering maps in singular cohomology, i.e., the elements of $\lim _{n} T_{n}^{R}(h, k)$, where $h(X)=H^{q}(X ; L)$ and $k(X)=H^{q}\left(X ; L^{\prime}\right)$, are determined by the transfers for 2 -fold ramified covering maps.

THEOREM 4.9. The function

$$
\iota: T_{n+1}^{R}\left(H^{q}(-; L), H^{q}\left(-; L^{\prime}\right)\right) \rightarrow T_{n}^{R}\left(H^{q}(-; L), H^{q}\left(-; L^{\prime}\right)\right)
$$

is an isomorphism for $n \geq 2(q>0)$.

Proof. Since $h=H^{q}(-; L)$, we have $\mathcal{H}=K(L, q)$, which is a CWcomplex. By [9], $\mathrm{SP}^{n} \mathcal{H}$ is also a CW-complex and a subcomplex of $\mathrm{SP}^{n+1} \mathcal{H}$. Therefore, we have a (H-coexact) cofibration sequence

$$
\mathrm{SP}^{n} \mathcal{H} \stackrel{i}{\hookrightarrow} \mathrm{SP}^{n+1} \mathcal{H} \stackrel{j}{\rightarrow} \mathrm{SP}^{n+1} \mathcal{H} / \mathrm{SP}^{n} \mathcal{H}
$$

Denote by $\widetilde{\mathrm{SP}}^{n+1} \mathcal{H}$ the reduced symmetric power of $\mathcal{H}$, i.e., the quotient of the action of $\Sigma_{n+1}$ on the smash product $\mathcal{H} \wedge \cdots \wedge \mathcal{H}(n+1$ factors $)$. Clearly, $\mathrm{SP}^{n+1} \mathcal{H} / \mathrm{SP}^{n} \mathcal{H} \approx \widetilde{\mathrm{SP}^{n+1}} \mathcal{H}$. Using the exact cohomology sequence of the cofibration sequence above for the theory $k(X)=H^{q}\left(X ; L^{\prime}\right)$ gives us an exact sequence 
$\widetilde{H}^{q}\left(\widetilde{\mathrm{SP}}^{n+1} \mathcal{H} ; L^{\prime}\right) \stackrel{j^{*}}{\rightarrow} H^{q}\left(\mathrm{SP}^{n+1} \mathcal{H} ; L^{\prime}\right) \stackrel{i^{*}}{\rightarrow} H^{q}\left(\mathrm{SP}^{n} \mathcal{H} ; L^{\prime}\right) \stackrel{\delta}{\rightarrow} \widetilde{H}^{q+1}\left(\widetilde{\mathrm{SP}^{n+1}} \mathcal{H} ; L^{\prime}\right)$, and Proposition 4.3 gives us the commutative diagram

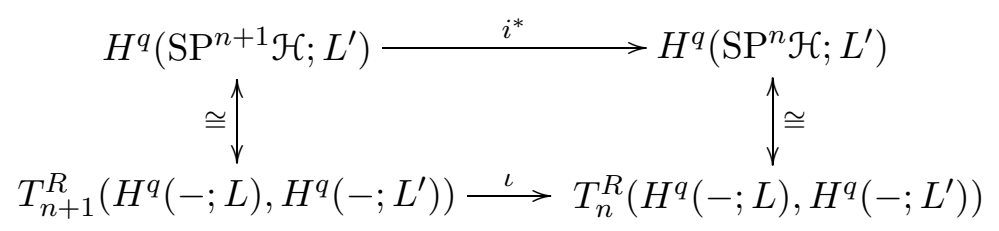

Since $\mathcal{H}=K(L, q)$ is $(q-1)$-connected, by [9], $\widetilde{\mathrm{SP}^{n+1}} \mathcal{H}$ is $(2 n-2+q)$ connected. Therefore, by the Hurewicz isomorphism theorem and the universal coefficient theorem, we see that $\widetilde{H}^{q}\left(\widetilde{\mathrm{SP}}^{n+1} \mathcal{H} ; L^{\prime}\right)$ and $\widetilde{H}^{q+1}\left(\widetilde{\mathrm{SP}^{n+1}} \mathcal{H} ; L^{\prime}\right)$ are both zero, provided that $n \geq 2$. Hence, $\iota$ is an isomorphism for $n \geq 2$.

In the case of theories other than the ordinary ones (given by EilenbergMac Lane spaces), there are nontrivial transfers. The following is an interesting case.

EXAMPLE 4.10. We analyze transfers for vector bundles. Let $\mathcal{H}$ be BU(1) and $\mathcal{K}$ be $\mathrm{BU}(k)$. We denote by $\operatorname{Vect}_{\mathbb{C}}^{k}(X)$ the set of isomorphism classes of numerable complex $k$-dimensional vector bundles over $X$. By 2.7, given an $n$-fold ramified covering map $p: E \rightarrow X$, there is a bijection between transfers $t_{p}: \operatorname{Vect}_{\mathbb{C}}^{1}(E) \rightarrow \operatorname{Vect}_{\mathbb{C}}^{k}(X)$ and elements in $\left[\operatorname{SP}^{n} \mathrm{BU}(1), \mathrm{BU}(k)\right] \cong$ $\operatorname{Vect}_{\mathbb{C}}^{k}\left(\mathrm{SP}^{n} \mathrm{BU}(1)\right)$.

Notice that $\mathrm{BU}(1)$ is a topological abelian group whose model can be defined by $\mathrm{BU}(1)=F\left(\mathbb{S}^{1}, \mathrm{U}(1)\right)$ (see [5]). This group structure is given in terms of line bundles by the tensor product. Since BU(1) is an EilenbergMac Lane space of type $K(\mathbb{Z}, 2)$, this group structure also corresponds to the group structure in $H^{2}(X ; \mathbb{Z})$, where one maps each line bundle to its first Chern class.

Consider the map $\nu: \mathrm{BU}(1)^{n} \rightarrow \mathrm{BU}(1)$ defined by the product above, and which corresponds to the bundle $\gamma^{1} \nabla \cdots \nabla \gamma^{1}$, where $\gamma^{1}$ is the universal line bundle. Since BU(1) is abelian, this map defines a map $\bar{\nu}: \mathrm{SP}^{n} \mathrm{BU}(1) \rightarrow$ $\mathrm{BU}(1)$. Now let $\varrho: \mathrm{BU}(1) \rightarrow \mathrm{BU}(k)$ be the classifying map of the Whitney sum $\gamma^{1} \oplus \cdots \oplus \gamma^{1}$ of $k$ copies of $\gamma^{1}$. Then the homotopy class of $\varrho \circ \bar{\nu}$ defines a transfer as mentioned above. To see that this transfer is not trivial, consider the diagonal map $d: \mathrm{BU}(1) \rightarrow \mathrm{BU}(1)^{n}$. Denote by $\lambda$ the tensor product $\gamma^{1} \otimes \cdots \otimes \gamma^{1}$ of $n$ copies of $\gamma^{1}$. Then the map $\varrho \circ \bar{\nu} \circ q \circ d=\varrho \circ \nu \circ d$, where $q: \mathrm{BU}(1)^{n} \rightarrow \mathrm{SP}^{n} \mathrm{BU}(1)$ is the projection, classifies the bundle $\lambda \oplus \cdots \oplus \lambda$ ( $k$ copies $)$. By the comments above, $\gamma^{1} \mapsto c_{1}\left(\gamma^{1}\right)$ yields an isomorphism, and $H^{2}(\mathrm{BU}(1) ; \mathbb{Z}) \cong \mathbb{Z}$, therefore $c_{1}(\lambda)=n c_{1}\left(\gamma^{1}\right) \neq 0$. Hence $c_{1}(\lambda \oplus \cdots \oplus \lambda)=c_{1}(\lambda)+\cdots+c_{1}(\lambda)=k n c_{1}\left(\gamma^{1}\right) \neq 0$, and so the transfer defined is not trivial. 
A modified version of the previous example is the following.

ExAmple 4.11. Let $p: E \rightarrow X$ be an $n$-fold ramified covering map with multiplicity function $\mu$, let $\pi: \xi \rightarrow E$ be a (numerable) $k$-dimensional complex vector bundle, and let $\lambda^{\xi}=\operatorname{det} \xi \rightarrow E$ denote its determinant line bundle. Consider the bundle $\tau_{n}^{p}(\xi) \rightarrow X$ whose fiber over $x \in X$ is given by

$$
\tau_{n}^{p}(\xi)_{x}=\underbrace{\lambda_{e_{1}}^{\xi} \otimes \cdots \otimes \lambda_{e_{1}}^{\xi}}_{\mu\left(e_{1}\right)} \otimes \cdots \otimes \underbrace{\lambda_{e_{r}}^{\xi} \otimes \cdots \otimes \lambda_{e_{r}}^{\xi}}_{\mu\left(e_{r}\right)},
$$

where $p^{-1}(x)=\left\{e_{1}, \ldots, e_{r}\right\}$ and $\lambda_{e_{j}}^{\xi}$ denotes the fiber of $\lambda^{\xi}$ over $e_{j} \in E$. The classifying map for this bundle is given by

$$
X \stackrel{\varphi_{p}}{\rightarrow} \mathrm{SP}^{n} E \stackrel{\mathrm{SP}^{n} f_{\lambda \xi}}{\longrightarrow} \mathrm{SP}^{n} \mathrm{BGL}(1, \mathbb{C}) \stackrel{\bar{\nu}}{\rightarrow} \mathrm{BGL}(1, \mathbb{C}),
$$

where $f_{\lambda \xi}$ is the classifying map of $\lambda^{\xi}$. Thus we have a transfer

$$
\tau_{n}^{p}: \operatorname{Vect}_{k}^{\mathbb{C}}(E)=[E, \operatorname{BGL}(k, \mathbb{C})] \rightarrow[X, \operatorname{BGL}(1, \mathbb{C})]=\operatorname{Vect}_{1}^{\mathbb{C}}(X) .
$$

To compute the group of transfers of the previous example, we need the following.

Lemma 4.12. Let $\operatorname{det}_{k}: \mathrm{U}(k) \rightarrow \mathrm{U}(1)$ be the determinant function. Then Bdet $_{k}: \mathrm{BU}(k) \rightarrow \mathrm{BU}(1)$ is such that [Bdet] $=c_{1}\left(\gamma^{k}\right)$, the first Chern class of the universal bundle, thus it is a generator of $H^{2}(\mathrm{BU}(k)) \cong \mathbb{Z}$.

Proof. Take $\mathrm{BU}(k)=F\left(\mathbb{S}^{1}, \mathrm{U}(k)\right)$. Then $\operatorname{Bdet}_{k}: F\left(\mathbb{S}^{1}, \mathrm{U}(k)\right) \rightarrow$ $F\left(\mathbb{S}^{1}, \mathrm{U}(1)\right)$ is given by $\operatorname{Bdet}_{k}(u)=\operatorname{det}_{k} \circ u$. Since $\mathrm{BU}(1)=F\left(\mathbb{S}^{1}, \mathrm{U}(1)\right)$ is a $K(\mathbb{Z}, 2)$, we take it to represent the second cohomology groups. If $k=1$, then $\operatorname{det}_{1}: \mathrm{U}(1) \rightarrow \mathrm{U}(1)$ is the identity, hence

$$
\left[\text { Bdet }_{1}\right]=[\mathrm{id}] \in H^{2}(\mathrm{BU}(1))=[\mathrm{BU}(1), \mathrm{BU}(1)],
$$

which is the generator, since we are dealing with Eilenberg-MacLane spaces, and we have

$$
[\mathrm{BU}(1), \mathrm{BU}(1)] \stackrel{\cong}{\rightarrow} \operatorname{Hom}\left(\pi_{2}(\mathrm{BU}(1)), \pi_{2}(\mathrm{BU}(1))\right) \cong \operatorname{Hom}(\mathbb{Z}, \mathbb{Z}) \cong \mathbb{Z}
$$

By induction, we assume that the homotopy class of $\operatorname{Bdet}_{k}: \mathrm{BU}(k) \rightarrow$ $\mathrm{BU}(1)$ is $c_{1}\left(\gamma^{k}\right)$, and consider $H^{2}(\mathrm{BU}(k+1)) \stackrel{\mathrm{B} i^{*}}{\rightarrow} H^{2}(\mathrm{BU}(k))$, where $i$ is the inclusion map. By naturality and stability of the Chern classes, $\mathrm{B} i^{*} c_{1}\left(\gamma^{k+1}\right)$ $=c_{1}\left(\gamma^{k}\right)$. Since these classes are the generators, $\mathrm{B} i^{*}$ is an isomorphism. Recalling that $i: \mathrm{U}(k) \rightarrow \mathrm{U}(k+1)$ maps a matrix $A$ to $\left(\begin{array}{ll}A & 0 \\ 0 & 1\end{array}\right)$, one has $\mathrm{B} i^{*}\left[\operatorname{Bdet}_{k+1}\right]=\left[\operatorname{Bdet}_{k}\right]$, since

$$
\operatorname{Bdet}_{k+1} \circ \mathrm{B} i: x \mapsto \operatorname{det}_{k+1} i u(x)=\operatorname{det}_{k+1}\left(\begin{array}{cc}
u(x) & 0 \\
0 & 1
\end{array}\right)=\operatorname{det}_{k} u(x) .
$$

Therefore, $\left[\operatorname{Bdet}_{k+1}\right]=c_{1}\left(\gamma^{k+1}\right)$ and thus it is a generator. 
We have the following result.

Theorem 4.13. $T^{R}\left(\operatorname{Vect}_{k}^{\mathbb{C}}(-), \operatorname{Vect}_{1}^{\mathbb{C}}(-)\right) \cong \mathbb{Z}$ and the family of transfers $\tau_{n}^{p}$ constructed in Example 4.11 constitute a generator.

Proof. First we analyze the situation for the transfers for $n$-fold ramified covering maps. By Corollary 2.10,

$$
T_{n}^{R}\left(\operatorname{Vect}_{k}^{\mathbb{C}}(-), \operatorname{Vect}_{1}^{\mathbb{C}}(-)\right) \cong H^{2}\left(\operatorname{SP}^{n} \operatorname{BGL}(k, \mathbb{C}) ; \mathbb{Z}\right)
$$

Without losing generality, we may write $\mathrm{U}(k)$ instead of $\mathrm{GL}(k, \mathbb{C})$. Making use of the fibration $\mathrm{U}(k-1) \hookrightarrow \mathrm{U}(k) \rightarrow \mathbb{S}^{2 k-1}$, one easily shows that $\pi_{1}(\mathrm{U}(k)) \cong \mathbb{Z}$ for $k \geq 1$. With this and the same arguments used in the proof of 2.13 , one has

$$
H^{2}\left(\mathrm{SP}^{n} \operatorname{BGL}(k, \mathbb{C}) ; \mathbb{Z}\right) \cong \mathbb{Z}
$$

Now, consider the inverse system

$$
\cdots \rightarrow H^{2}\left(\mathrm{SP}^{3} \mathrm{BU}(k)\right) \rightarrow H^{2}\left(\mathrm{SP}^{2} \mathrm{BU}(k)\right) \rightarrow H^{2}(\mathrm{BU}(k)) .
$$

To show that all arrows are isomorphisms, take the cofiber sequence $\operatorname{SP}^{n} X \hookrightarrow$ $\mathrm{SP}^{n+1} X \rightarrow \overline{\mathrm{SP}}^{n+1} X=\mathrm{SP}^{n+1} X / \mathrm{SP}^{n} X$. By [9] we know that if $X$ is $(l-1)-$ connected, then $\overline{\mathrm{SP}}^{n+1} X$ is $(2 n+l-2)$-connected. Therefore, since $\mathrm{BU}(k)$ is 1-connected, in the exact sequence

$$
\widetilde{H}^{2}\left(\overline{\mathrm{SP}}^{n+1} \mathrm{BU}(k)\right) \rightarrow \widetilde{H}^{2}\left(\mathrm{SP}^{n+1} \mathrm{BU}(k)\right) \stackrel{\cong}{\rightarrow} \widetilde{H}^{2}\left(\mathrm{SP}^{n} \mathrm{BU}(k)\right) \rightarrow \widetilde{H}^{3}\left(\overline{\mathrm{SP}}^{n+1} \mathrm{BU}(k)\right)
$$

the middle arrow is an isomorphism if $n \geq 2$. In order to see that the last arrow on the right in the inverse system (4.14) is also an isomorphism, we do the following. Take $\mathrm{BU}(k)=F\left(\mathbb{S}^{1}, \mathrm{U}(k)\right)$, and take as base point $*$ the function given by $*(s)=\mathbf{1}$, the identity matrix, for all $s \in \mathbb{S}^{1}$. In particular, $\mathrm{BU}(1)$ is a topological abelian group. On the other hand, the inclusions $\mathrm{SP}^{n} \mathrm{BU}(k) \hookrightarrow \mathrm{SP}^{n+1} \mathrm{BU}(k)$ are given by $\left\langle u_{1} \ldots, u_{n}\right\rangle \mapsto\left\langle u_{1} \ldots, u_{n}, *\right\rangle$. $\mathrm{By}$ Lemma 4.12, the generator of $H^{2}(\mathrm{BU}(k))=[\mathrm{BU}(k), \mathrm{BU}(1)]$ as an infinite cyclic group is given by [Bdet]; on the other hand, the homotopy classes of the maps $\beta_{n}$ given by the diagrams

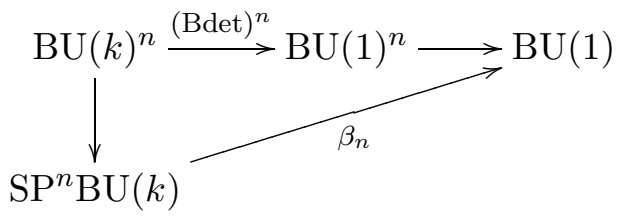

seen as elements in $\widetilde{H}^{2}\left(\mathrm{SP}^{n} \mathrm{BU}(k)\right)$, where the top arrow on the right-hand side is given by the abelian multiplication in $\mathrm{BU}(1)$, obviously map to each other in the inverse system (4.14). In particular, $\left[\beta_{2}\right] \mapsto\left[\beta_{1}\right]=[$ Bdet $]$. So, 
the last arrow on the right of the inverse system is surjective, and thus it is an isomorphism. Hence, all arrows are isomorphisms and the elements $\left[\beta_{n}\right]$ are generators of the infinite cyclic groups, and since each $\tau_{n}$ corresponds to $\left[\beta_{n}\right]$, the family $\tau=\left\{\tau_{n}\right\}$ is a transfer for ramified covering maps. Consequently, $T^{R}\left(\operatorname{Vect}_{k}^{\mathbb{C}}(-), \operatorname{Vect}_{1}^{\mathbb{C}}(-)\right) \cong \lim _{n} H^{2}\left(\operatorname{SP}^{n} \mathrm{BU}(k)\right) \cong \mathbb{Z}$, and $\tau$ is the generator.

5. Transfers in 1-dimensional integral cohomology. We consider $\left(H^{1}(-; \mathbb{Z}), H^{1}(-; \mathbb{Z})\right)$-transfers for $n$-fold ramified covering maps as well as for $n$-fold covering maps. We denote by $\Sigma_{n}$ \ $\mathbb{Z}$ the wreath product of $\Sigma_{n}$ and $\mathbb{Z}$, i.e., the semidirect product of $\Sigma_{n}$ with $\mathbb{Z}^{n}$, where $\Sigma_{n}$ acts on $\mathbb{Z}^{n}$ by permuting the summands. Therefore, the product in $\Sigma_{n} \prec \mathbb{Z}$ is given by $\left(\sigma, a_{1}, \ldots, a_{n}\right) \cdot\left(\tau, b_{1}, \ldots, b_{n}\right)=\left(\sigma \tau, a_{\tau(1)}+b_{1}, \ldots, a_{\tau(n)}+b_{n}\right)$.

LEMma 5.1. The following hold:

(a) $\mathrm{B}\left(\Sigma_{n} \curlywedge \mathbb{Z}\right)=E \Sigma_{n} \times \Sigma_{n}(\mathbb{R} / \mathbb{Z})^{n}$.

(b) Let $f: \Sigma_{n} \backslash \mathbb{Z} \rightarrow \mathbb{Z}$ be the homomorphism defined by $f\left(\sigma, a_{1}, \ldots, a_{n}\right)=$ $a_{1}+\cdots+a_{n}$. Then $\mathrm{B} f: \mathrm{B}\left(\Sigma_{n} \imath \mathbb{Z}\right) \rightarrow \mathrm{B} \mathbb{Z}$ is given by

$\varphi: E \Sigma_{n} \times_{\Sigma_{n}}(\mathbb{R} / \mathbb{Z})^{n} \rightarrow \mathbb{R} / \mathbb{Z}$, where $\varphi\left\langle y, \bar{r}_{1}, \ldots, \bar{r}_{n}\right\rangle=\bar{r}_{1}+\cdots+\bar{r}_{n}$.

Proof. (a) Consider the space $E \Sigma_{n} \times \mathbb{R}^{n}$. This space is contractible and has a free action of $\Sigma_{n} \imath \mathbb{Z}$ given by

$$
\left(y, r_{1}, \ldots, r_{n}\right) \cdot\left(\sigma, a_{1}, \ldots, a_{n}\right)=\left(y \cdot \sigma, r_{\sigma(1)}+a_{1}, \ldots, r_{\sigma(n)}+a_{n}\right) .
$$

Therefore, $\mathrm{B}\left(\Sigma_{n} \imath \mathbb{Z}\right)=\left(E \Sigma_{n} \times \mathbb{R}^{n}\right) /\left(\Sigma_{n} \prec \mathbb{Z}\right)$. Now consider the following diagram:

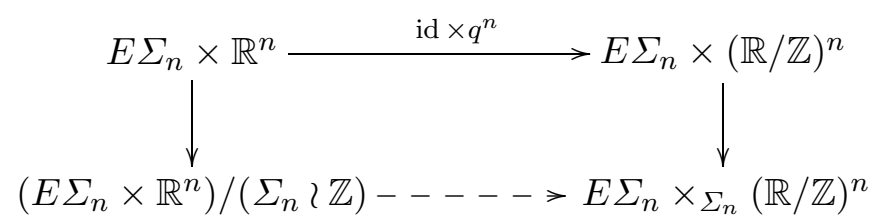

Since the quotient map $q: \mathbb{R} \rightarrow \mathbb{R} / \mathbb{Z}$ is a covering map, so also is id $\times q^{n}$; in particular, it is a quotient map as are the two vertical maps. Thus they clearly define a homeomorphism $\left(E \Sigma_{n} \times \mathbb{R}^{n}\right) /\left(\Sigma_{n} \imath \mathbb{Z}\right) \rightarrow E \Sigma_{n} \times \Sigma_{n}(\mathbb{R} / \mathbb{Z})^{n}$ given by $\left\langle y, r_{1}, \ldots, r_{n}\right\rangle \mapsto\left\langle y, \bar{r}_{1}, \ldots, \bar{r}_{n}\right\rangle$.

(b) Consider the action $\Sigma_{n} \imath \mathbb{Z} \times \mathbb{Z} \rightarrow \mathbb{Z}$ given by

$$
\left(\sigma, a_{1}, \ldots, a_{n}\right) \cdot k=f\left(\sigma, a_{1}, \ldots, a_{n}\right)+k=a_{1}+\cdots+a_{n}+k .
$$

By part (a), we have a principal $\Sigma_{n} \prec \mathbb{Z}$-bundle $p: E \Sigma_{n} \times \mathbb{R}^{n} \rightarrow E \Sigma_{n} \times \Sigma_{n}$ $(\mathbb{R} / \mathbb{Z})^{n}$, where $p\left(y, r_{1}, \ldots, r_{n}\right)=\left\langle y, \bar{r}_{1}, \ldots, \bar{r}_{n}\right\rangle$. Then $\mathrm{B} f$ classifies the associated principal $\mathbb{Z}$-bundle $\bar{p}:\left(E \Sigma_{n} \times \mathbb{R}^{n}\right) \times \Sigma_{n} \mathbb{Z} \mathbb{Z} \rightarrow E \Sigma_{n} \times \Sigma_{n}(\mathbb{R} / \mathbb{Z})^{n}$. 
Now consider the following diagram:

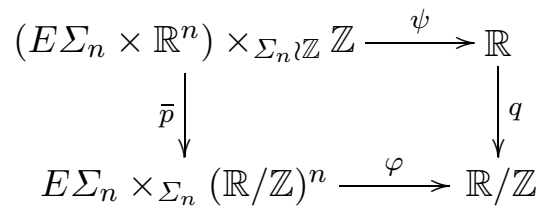

where $\psi\left\langle\left(y, r_{1}, \ldots, r_{n}\right), k\right\rangle=r_{1}+\cdots+r_{n}+k$. Clearly this is a morphism of principal $\mathbb{Z}$-bundles; therefore, $\mathrm{B} f \simeq \varphi$.

LEMMA 5.2. $H^{1}\left(E \Sigma_{n} \times_{\Sigma_{n}} K(\mathbb{Z}, 1)^{n} ; \mathbb{Z}\right) \cong \mathbb{Z}$.

Proof. Since $K(\mathbb{Z}, 1)=\mathrm{B} \mathbb{Z}$ and $E \Sigma_{n} \times \Sigma_{n} \mathrm{~B} \mathbb{Z}^{n}=\mathrm{B}\left(\Sigma_{n} \prec \mathbb{Z}\right)$ by 5.1 , we have

$$
H^{1}\left(E \Sigma_{n} \times_{\Sigma_{n}} K(\mathbb{Z}, 1)^{n} ; \mathbb{Z}\right) \cong H^{1}\left(\mathrm{~B}\left(\Sigma_{n} \prec \mathbb{Z}\right) ; \mathbb{Z}\right) \cong \operatorname{Hom}\left(\Sigma_{n} \prec \mathbb{Z}, \mathbb{Z}\right) .
$$

Let $\iota: \mathbb{Z}^{n} \hookrightarrow \Sigma_{n} \imath \mathbb{Z}$ be the inclusion given by $\iota\left(a_{1}, \ldots, a_{n}\right)=\left(1, a_{1}, \ldots, a_{n}\right)$, and consider $\iota^{*}: \operatorname{Hom}\left(\Sigma_{n} \imath \mathbb{Z}, \mathbb{Z}\right) \rightarrow \operatorname{Hom}\left(\mathbb{Z}^{n}, \mathbb{Z}\right)$. Let $F: \Sigma_{n} \imath \mathbb{Z} \rightarrow \mathbb{Z}$ be a homomorphism and assume that $\iota^{*}(F)=0$. Then $F\left(1, a_{1}, \ldots, a_{n}\right)=0$ for all $\left(a_{1}, \ldots, a_{n}\right) \in \mathbb{Z}^{n}$. Since any element $\left(\sigma, a_{1}, \ldots, a_{n}\right) \in \Sigma_{n}$ 々 $\mathbb{Z}$ can be written as $\left(\sigma, a_{1}, \ldots, a_{n}\right)=(\sigma, 0, \ldots, 0) \cdot\left(1, a_{1}, \ldots, a_{n}\right)$, we see that $F\left(\sigma, a_{1}, \ldots, a_{n}\right)=F(\sigma, 0, \ldots, 0)+F\left(1, a_{1}, \ldots, a_{n}\right)=F(\sigma, 0, \ldots, 0)$. But $\sigma$ is an element of $\Sigma_{n}$, which is a finite subgroup of $\Sigma_{n} \prec \mathbb{Z}$, and the codomain is free, hence $F(\sigma, 0, \ldots, 0)=0$. Therefore, $F=0$, so that $\iota^{*}$ is a monomorphism.

Let $e_{i}$ be the element in $\mathbb{Z}^{n}$ whose coordinates are all zero, except the $i$ th one that is equal to 1 . Let $\tau \in \Sigma_{n}$ be the permutation given by $\tau(i)=j$ and $\tau(k)=k$ for $k \neq i, j$. Then $\left(1, e_{i}\right) \cdot(\tau, 0)=\left(\tau, e_{j}\right)=(\tau, 0) \cdot\left(1, e_{j}\right)$. Hence $F\left(\left(1, e_{i}\right) \cdot(\tau, 0)\right)=F\left((\tau, 0) \cdot\left(1, e_{j}\right)\right)$. But $F\left(\left(1, e_{i}\right) \cdot(\tau, 0)\right)=F\left(1, e_{i}\right)$ and $F\left((\tau, 0) \cdot\left(1, e_{j}\right)\right)=F\left(1, e_{j}\right)$. Therefore, $F\left(1, e_{i}\right)=F\left(1, e_{j}\right)$. Since we have an isomorphism $\psi: \operatorname{Hom}\left(\mathbb{Z}^{n}, \mathbb{Z}\right) \cong \mathbb{Z}^{n}$ given by $\psi(f)=\left(f\left(e_{1}\right), \ldots, f\left(e_{n}\right)\right)$, it follows that $\operatorname{im}\left(\psi \circ \iota^{*}\right)$ is the diagonal subgroup in $\mathbb{Z}^{n}$, isomorphic to $\mathbb{Z}$. Moreover, the canonical element $\Omega$ in $\operatorname{Hom}\left(\Sigma_{n} \mathfrak{Z}, \mathbb{Z}\right)$ given by $\Omega\left(\sigma, a_{1}, \ldots, a_{n}\right)=$ $a_{1}+\cdots+a_{n}$ is a generator because $\psi \iota^{*}(\Omega)=\left(\iota^{*}(\Omega)\left(e_{1}\right), \ldots, \iota^{*}(\Omega)\left(e_{n}\right)\right)=$ $(1, \ldots, 1)$.

As a consequence of Lemma 5.2 , the $\left(H^{1}(-; \mathbb{Z}), H^{1}(-; \mathbb{Z})\right)$-transfers for $n$-fold ramified covering maps coincide with the $\left(H^{1}(-; \mathbb{Z}), H^{1}(-; \mathbb{Z})\right)$-transfers for $n$-fold ordinary covering maps. Moreover, there is exactly one transfer for each integer. In other words, we have the following.

THEOREM 5.3. The restriction

$$
r: T_{n}^{R}\left(H^{1}(-; \mathbb{Z}), H^{1}(-; \mathbb{Z})\right) \rightarrow T_{n}\left(H^{1}(-; \mathbb{Z}), H^{1}(-; \mathbb{Z})\right)
$$

is an isomorphism, and both groups are isomorphic to $\mathbb{Z}$. 
Proof. By 3.3, we have a commutative diagram

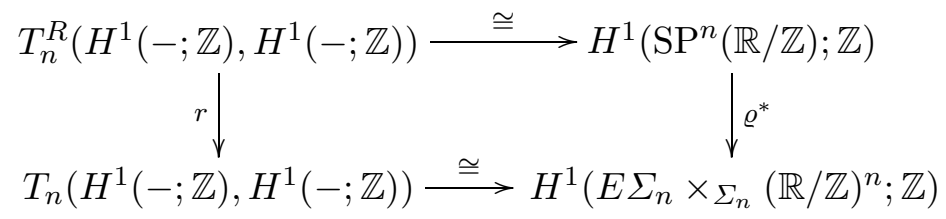

By $[2,5.2 .23]$, the canonical inclusion $j: \mathbb{R} / \mathbb{Z} \hookrightarrow \mathrm{SP}^{n}(\mathbb{R} / \mathbb{Z})$ is a homotopy equivalence. Let $w: \mathrm{SP}^{n}(\mathbb{R} / \mathbb{Z}) \rightarrow \mathbb{R} / \mathbb{Z}$ be the map defined by $w\left\langle\bar{r}_{1}, \ldots, \bar{r}_{n}\right\rangle$ $=\bar{r}_{1}+\cdots+\bar{r}_{n}$. Then $j^{*}[w]=[w \circ j]=[\mathrm{id}]$, which is a generator of $H^{1}(\mathbb{R} / \mathbb{Z} ; \mathbb{Z})$. Therefore, $H^{1}\left(\mathrm{SP}^{n}(\mathbb{R} / \mathbb{Z}) ; \mathbb{Z}\right) \cong \mathbb{Z}$, with generator given by $[w]$.

By Lemma 5.1(a),

$$
H^{1}\left(E \Sigma_{n} \times \Sigma_{n}(\mathbb{R} / \mathbb{Z})^{n} ; \mathbb{Z}\right) \cong H^{1}\left(\mathrm{~B}\left(\Sigma_{n} \curlywedge \mathbb{Z}\right) ; \mathbb{Z}\right)=\left[\mathrm{B}\left(\Sigma_{n} \curlywedge \mathbb{Z}\right), \mathrm{B} \mathbb{Z}\right] .
$$

By $[2,6.4 .6]$,

$$
\left[\mathrm{B}\left(\Sigma_{n} \prec \mathbb{Z}\right), \mathrm{B} \mathbb{Z}\right] \cong \operatorname{Hom}\left(\pi_{1}\left(\mathrm{~B}\left(\Sigma_{n} \prec \mathbb{Z}\right)\right), \pi_{1}(\mathrm{~B} \mathbb{Z})\right)=\operatorname{Hom}\left(\Sigma_{n} \imath \mathbb{Z}, \mathbb{Z}\right),
$$

and by Lemma $5.2, \operatorname{Hom}\left(\Sigma_{n} \imath \mathbb{Z}, \mathbb{Z}\right) \cong \mathbb{Z}$, with a generator $f: \Sigma_{n} \imath \mathbb{Z} \rightarrow \mathbb{Z}$ defined by $f\left(\sigma, a_{1}, \ldots, a_{n}\right)=a_{1}+\cdots+a_{n}$. Therefore, by the naturality of the homotopy equivalence $\Omega \mathrm{B} G \simeq G$ for any discrete group $G$, a generator of $H^{1}\left(\mathrm{~B}\left(\Sigma_{n} \imath \mathbb{Z}\right) ; \mathbb{Z}\right)$ is given by B $f$. By Lemma 5.1(b), B $f \simeq \varphi$. Since $\varrho^{*}[w]=$ $[w \circ \varrho]$, and $w \varrho\left\langle y, \bar{r}_{1}, \ldots, \bar{r}_{n}\right\rangle=w\left\langle\bar{r}_{1}, \ldots, \bar{r}_{n}\right\rangle=\bar{r}_{1}+\cdots+\bar{r}_{n}$, we have $w \circ \varrho=\varphi$. Therefore, $\varrho^{*}$ is an isomorphism and hence so is $r$.

As an immediate consequence we have the following.

COROLlaRY 5.4. There is an isomorphism $T_{n}^{R}\left(H^{1}(-; \mathbb{Z}), H^{1}(-; \mathbb{Z})\right) \rightarrow \mathbb{Z}$. The canonical transfert, as given in 1.4 , corresponds to $1 \in \mathbb{Z}$. For any other integer $k$ the corresponding transfer is ${ }_{k} t$ given by $\left({ }_{k} t\right)_{n}^{p}(\eta)=k \cdot t_{n}^{p}(\eta)$ for any $n$-fold ramified covering map $p: E \rightarrow X$ and any element $\eta \in H^{1}(E ; \mathbb{Z})$.

\section{References}

[1] J. F. Adams, Infinite Loop Spaces, Ann. of Math. Stud. 90, Princeton Univ. Press, Princeton, NJ, 1978.

[2] M. Aguilar, S. Gitler and C. Prieto, Algebraic Topology from a Homotopical Viewpoint, Universitexts, Springer, New York, 2002.

[3] M. Aguilar and C. Prieto, Transfers for ramified covering maps in homology and cohomology, to appear.

[4] P. J. Huber, Homotopical cohomology and Čech cohomology, Math. Ann. 144 (1961), $73-76$.

[5] M. C. McCord, Classifying spaces and infinite symmetric products, Trans. Amer. Math. Soc. 146 (1969), 273-298.

[6] F. W. Roush, Transfer in Generalized Cohomology Theories, Akadémiai Kiadó, Budapest, 1999.

[7] L. Smith, Transfer and ramified coverings, Math. Proc. Cambridge Philos. Soc. 93 (1983), 485-493. 
[8] N. Steenrod, Cohomology operations, and obstructions to extending continuous functions, Adv. Math. 8 (1972), 371-416.

[9] P. J. Welcher, Symmetric products and the stable Hurewicz homomorphism, Illinois J. Math. 24 (1980), 527-544.

[10] G. W. Whitehead, Elements of Homotopy Theory, Grad. Texts in Math. 61, Springer, New York, 1978.

Instituto de Matemáticas

UNAM

04510 México, D.F., Mexico

E-mail: marcelo@math.unam.mx

cprieto@math.unam.mx

Received 4 December 2004;

in revised form 7 November 2005 